NASH/TM- 1998- 207321

\title{
AIAA 98-0471 \\ Design and Use of Microphone \\ Directional Arrays for Aeroacoustic Measurements
}

William M. Humphreys, Jr.

Thomas F. Brooks

William W. Hunter, Jr.

Kristine R. Meadows

NASA Langley Research Center Hampton, VA 23681-0001

\section{6st Aerospace Sciences}

Meeting \& Exhibit

January 12-15, 1998 / Reno, NV

For permission to copy or republish, contact the American Institute of Aeronautics and Astronautics 1801 Alexander Bell Drive, Suite 500, Reston, Virginia 20191-4344 


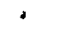


AIAA-98-0471

\title{
DESIGN AND USE OF MICROPHONE DIRECTIONAL ARRAYS FOR AEROACOUSTIC MEASUREMENTS
}

\author{
William M. Humphreys, Jr." \\ Thomas F. Brooks ${ }^{\dagger}$ \\ William W. Hunter, Jr. ${ }^{\ddagger}$ \\ Kristine R. Meadows' \\ Fluid Mechanics and Acoustics Division
NASA Langley Research Center
Hampton, Virginia 23681-0001
}

\section{Abstract}

An overview of the development of two microphone directional arrays for aeroacoustic testing is presented. These arrays were specifically developed to measure airframe noise in the NASA Langley Quiet Flow Facility. A large aperture directional array using 35 flush-mounted microphones was constructed to obtain high resolution noise localization maps around airframe models. This array possesses a maximum diagonal aperture size of 34 inches. A unique logarithmic spiral layout design was chosen for the targeted frequency range of $2-30 \mathrm{kHz}$. Complementing the large array is a small aperture directional array, constructed to obtain spectra and directivity information from regions on the model. This array, possessing 33 microphones with a maximum diagonal aperture size of 7.76 inches, is easily moved about the model in elevation and azimuth. Custom microphone shading algorithms have been developed to provide a frequency- and position-invariant sensing area from $10-40 \mathrm{kHz}$ with an overall targeted frequency range for the array of $5-60 \mathrm{kHz}$. Both arrays are employed in acoustic measurements of a 6 percent of full scale airframe model consisting of a main element NACA $63_{2}-215$ wing section with a 30 percent chord half-span

*Research Scientish, Measurement Science and Technology Branch, Senior Member AIAA.

'Senior Research Scientist, Aeroacoustios Branch, Associale Fellow AIAA

${ }^{ \pm}$Senior Research Scientish, Measurement Science and Technology Branch.

'Research Scientist, Aeroacoustics Branch, Member AIAA.

Copyright $O 1998$ by the American Institute of Aeronautics and Astronauties, Inc. No copyright is ascerted in the United States under Title 17, U.S. Code. The U.S. Government has a royalty-free license to exercise all rights under the copyright claimed herein for government purposes. All other rights are reserved by the copyright owner. flap. Representative data obtained from these measurements is presented, along with details of the array calibration and data post-processing procedures.

\section{Nomenclature}

Superscript $o \quad$ source location

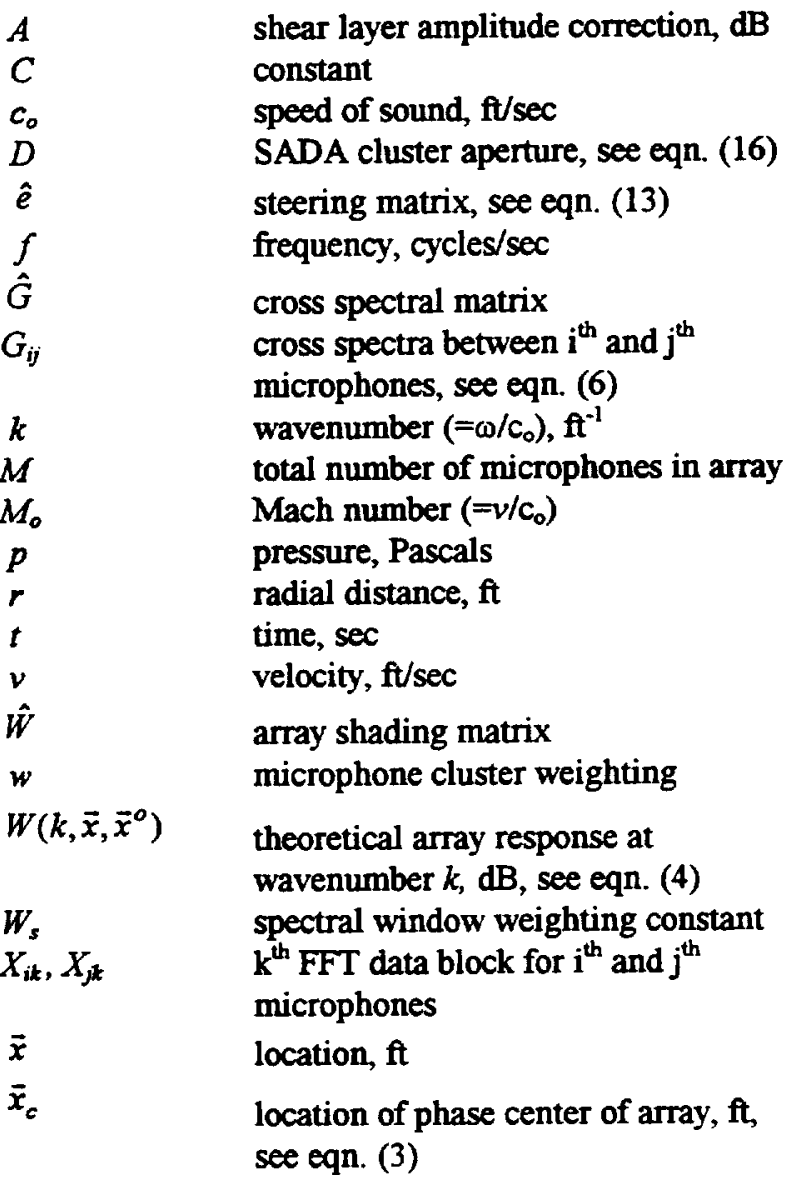


acoustic wavelength, $\mathrm{ft}$

SADA array weighting control

frequency, $\mathrm{rad} / \mathrm{sec}$

shear layer phase correction for $\omega$, radians, see eqns. (9) and (13)

\section{Introduction}

Over the past several years a growing need has emerged for accurate and robust noise measurement instrumentation in aerospace research facilities. This need is partly driven by research programs such as the NASA Advanced Subsonic Technology (AST) Program, which has set as one of its goals the achieving of a greater than $10 \mathrm{~dB}$ reduction in total aircraft effective perceived noise by the year 2000 (referenced to levels measured in 1992). This goal requires the collection of experimental databases of various noise generation mechanisms from which accurate and efficient noise prediction tools can be developed to guide noise reduction design. Recently, emphasis has been placed on the measurement and modeling of airframe noise, defined as the nonpropulsive component of aircraft noise which is due to unsteady flow about the airframe components (flaps, slats, undercarriage, etc.).

One of the databases desired by computational airframe noise modelers is farfield noise data measured on various baseline and modified aircraft components. Traditional single microphone measurements of this noise have been hampered by poor signal-to-noise characteristics, spurring the development of a variety of new measurement techniques. Early techniques employed the concept of an "acoustic mirror", where a large concave elliptic mirror and an associated microphone were positioned in the acoustic far field. ${ }^{1-3}$ Such mirrors were capable of locating individual sound sources accurately, but suffered the drawback of requiring mechanical movement to determine source distributions around models. The mirrors also became excessively large when measurements of lower frequencies $(<2 \mathrm{kHz}$ ) were required. Nevertheless, such mirrors continue to have applicability in some of the larger research facilities. ${ }^{4}$

In addition to acoustic mirrors, distributions of individual microphones have been employed to determine airframe noise source characteristics. In particular, such systems have proven valuable in the understanding of single-element airfoil self noise. ${ }^{5-6}$ While not strictly considered a directional array (the outputs of all microphones were not combined as in beamforming), such systems capitalized on the amplitude and phase relationships between clusters of microphones. As such, they can be considered one of the precursors to the current generation of microphone directional arrays.

Modern microphone directional arrays for aeroacoustic research have as their origin early radio and radar antenna arrays and U.S. Navy hydrophone arrays (used for the detection of submarines as early as World War II). ${ }^{7.8}$ Soderman and Noble were among the first researchers to adapt this earlier work for aeroacoustics when they constructed a one-dimensional end-fire array to evaluate jet noise in the NASA Ames 40- by 80 -foot Wind Tunnel. ${ }^{9-10}$ At the same time Billingsley and Kinns constructed a one-dimensional linear array of microphones for real-time sound source location on full-size jet engines. " More recently such directional arrays have been extended to include twodimensional microphone layouts with the work of Brooks, Marcolini and Pope ${ }^{12-13}$, Underbrink and Dougherty $^{14}$, and Watts and Mosher. ${ }^{15-16}$

Two different state-of-the-art, two-dimensional microphone directional arrays are described in this paper. These are designed to provide broadband source localization and directivity information needed to characterize airframe noise and noise reduction concepts. Both arrays have been successfully used by the authors to obtain data for a wing / flap model. ${ }^{17}$ This paper expands on the previous work by providing detailed descriptions of the design and construction of the two directional arrays. The philosophy surrounding their design as well as development of unique data processing algorithms to allow accurate noise spectra and source images to be obtained are discussed. Finally, several representative examples of data collected with the instruments are illustrated.

\section{Directional Array Development}

\section{Concept}

The basic principle of a microphone directional array can be simply illustrated. Assume a simple monochromatic acoustic point source is located in quiescent space at location $\vec{x}$ (see Figure 1). A solution for $r>0$ representing the propagation of $a$ pressure wave radially in all directions is given by

$$
p(r, t)=\frac{C}{r} e^{j(\omega t-k r)}
$$

where $C$ is a constant, $r$ is the radial distance from the source origin, $\omega$ is the frequency of the wave, and $k$ is the corresponding wavenumber. Assume now that an array of $\mathrm{M}$ microphones is placed a finite distance from 
the source. Each microphone senses a slightly different phase-shifted waveform depending on its distance from the source. The pressure $p_{m}(t)$ measured at the $\mathrm{m}$-th microphone is denoted as

$$
p_{m}(t)=\frac{C}{r_{m}} e^{j \omega\left(t-\frac{m}{c_{0}}\right)}
$$

where $r_{m}$ represents the distance from the location to the $\mathrm{m}$-th microphone. The $\left(t-r_{m} / c_{0}\right)$ term is the retarded time from the source to the microphone. In order to focus on a source, the individual microphone outputs can be phase shifted an amount equal to their propagation delay and then summed together (or stacked). This yields a single output signal for the array in a process commonly referred to as delay-andsum beamforming. By adjusting the propagation delays, one is able to electronically steer the array to points in space, selecting regions of interest to ascertain noise production while providing noise rejection not found in individual microphone measurements. This steering can provide the same capability as the earlier acoustic mirror techniques but without the necessity of physically moving the array to measure source distributions.

\section{Array Response}

The phase center of the array is defined as ${ }^{18}$

$$
\vec{x}_{c}=\frac{1}{M} \sum_{m=1}^{M} \vec{x}_{m}
$$

Using this, the ideal array response for a simple source can be expressed as

$$
W\left(k, \vec{x}, \bar{x}^{o}\right) \equiv \sum_{m=1}^{M} w_{m} \frac{r^{o}}{r_{m}^{o}} e^{j k\left[\left(r^{\circ}-r\right)-\left(r_{m}^{o}-r_{m}\right)\right]}
$$

where $\vec{x}$ is an arbitrary Cartesian location in space to which the array is electronically steered, $\vec{x}^{\circ}$ is the source location, $r^{\circ}$ and $r_{m}{ }^{\circ}$ are the distances from the source to $\vec{x}_{c}$ and the $\mathrm{m}$-th microphone, respectively, and $r$ and $r_{m}$ are the distances from the steering location to $\vec{x}_{c}$ and the microphone. The term $w_{m}$ represents a microphone weighting factor which can be used to modify the array response.

The array response is normally expressed in decibels referenced to the level obtained at $\vec{x}^{\circ}$ :

$$
d B(\vec{x})=20 \log _{10}\left[\frac{\left|W\left(k, \bar{x}, \vec{x}^{o}\right)\right|}{\left|W\left(k, \vec{x}^{o}, \vec{x}^{\circ}\right)\right|}\right]
$$

This response is plotted as a contour map with contour level proportional to $d B(\vec{x})$, representing the computation of equation (5) over a large number of steering locations lying on a surface a finite distance from the array. Such plots represent the spatial filtering of the array graphically at wavenumber $k$, and allows one to examine the beamwidth and lobe structure.

\section{Array Design Criteria for Airframe \\ Noise Measurements}

Test Model and Facility: The test program is intended to investigate the mechanisms of sound generation on high-lift wing configurations. In Figure 2, the test model apparatus and the Large Aperture Directional Array, to be discussed, are shown mounted in the Langley Quiet Flow Facility (QFF). The QFF is a quiet open-jet facility designed specifically for anechoic acoustic testing. ${ }^{19}$ For the present airframe model testing, a 2 by 3-foot rectangular open-jet nozzle is employed. The model is a NACA $63_{2}-215$ main element airfoil with a 30 percent chord half-span Fowler flap. In the photo, the model is visible through the Plexiglas windows located on the side plates. The model section is approximately 6 percent of a full-scale configuration, with a main element chord length of 16 inches, a flap chord length of 4.5 inches, and a full span of 36 inches. The main element and flap are fully instrumented with static pressure ports and unsteady pressure transducers. To hold the model in place, the vertical side plates are fastened rigidly to the side plate supports of the nozzle. Appropriate acoustic foam treatments are applied to all edges and supports to reduce acoustic reflections from these surfaces. More model and facility details can be found in Reference 17.

Array Design Criteria: In choosing an array design, specifically the microphone layout with respect to the noise source to be studied, one must be aware of the character of the source distributions. The basic delay-and-sum beamformer procedure, described above, renders an array output which assumes any single source to be an omni-directional simple monopole, or any distribution of sources to be that of incoherent (uncorrelated) simple monopoles. But, when the sources are multi-pole and/or coherent over a 
spatial source distribution, the noise is not omnidirectional. For such directional sources, variations in noise field coherence, amplitude, and phase can occur over the face of the array. Major difficulties occur when the directivity has an oscillatory sweeping or rotational phase behavior. Still, even when source directivity is stationary, which is assumed to be the case for the airframe noise problem, spatial variations can cause moderate to severe errors in source amplitude, resolution, and localization. This is because the phase variations are interpreted as retarded time delays, and amplitude/coherence variations modify the relative contribution of each microphone to the array output. Indeed, the effect of limited spatial coherence for the noise directivity is to effectively break an array with too large an aperture (overall width of the array of microphones) into a group of smaller sub-arrays whose individual steered output spectra are summed together. This is "pressure-squared" summing rather than the desired "linear-pressure" summing (operation indicated by equation (4)), thereby modifying the desired "design" characteristics of the array. To avoid such errors, all array microphones should be placed within approximately the same source directivity (producing generally a small array), where amplitude and phase appear as if the source were omni-directional. However, as will be seen, this design constraint cannot always be fully met and still have the desired array resolution at the frequencies of interest.

Airframe noise measurements present an array design challenge in that not only is source directivity information required (necessitating the use of a small aperture array to satisfy the concerns discussed above), but also accurate localization of the source distributions is desired down to the order of the smallest wavelength of interest, typically one to two tenths of an inch. This latter need requires that the array aperture be large in order to minimize the array beamwidth, defined here as the width across the main response lobe over which the sensing level is within a given $\mathrm{dB}$ level from the peak level. However, the required spectra and directivity information dictate the use of a small array aperture to ensure that all microphones are at approximately the same directivity angle.

It was decided to address the two conflicting aperture requirements through the construction of two array designs. A Large Aperture Directional Array (LADA) was designed to produce high spatial resolution (narrow beamwidth) noise source localization maps over a defined surface on the model. To obtain quantitative spectra and directivity information, particularly for the dominant noise sources identified with the LADA, a Small Aperture Directional Array (SADA) was also designed. This array was constructed to be movable about the model in both elevation and azimuth, as opposed to the LADA which was fixed in location. The SADA results can also be used to evaluate the degree of directivity uniformity the LADA encounters to add confidence to the LADA results.

\section{Description of Two Directional Arrays}

Large Aperture Directional Array (LADA): The LADA is shown to the left in Figure 2, on the pressure side of the model, positioned 4 feet from the mid-span of the airfoil main element trailing edge. A 4-foot diameter fiberglass panel provides a flat surface to flush mount all microphones. The panel is attached to a pan-tilt unit secured to a rigid tripod support. This allows precise alignment changes in the elevation and azimuth of the face of the array. A small laser diode pointer is place at $\vec{x}_{c}$, corresponding to the center of the fiber glass panel. The LADA incorporates $35 \mathrm{~B} \& \mathrm{~K}$ model 4135 , 1/4-inch microphones placed in a twodimensional pattern consisting of logarithmic spirals. The microphone layout, shown in Figure 3, consists of five spirals of seven microphones each with the innermost microphones lying on a 1 -inch radius and the outer-most on a 17 -inch radius. The locations of the microphones, viewed from the front of the array, are listed in Table 1. This design is very similar to a multi-arm logarithmic spiral array with linearly spaced spiral elements described in Reference 14. This design results in acceptable beamwidth and peak sidelobe height over a targeted design frequency range of 2-30 kHz.

Figure 4 shows a series of contour plots showing LADA array responses using equations (4) and (5) for $6,10,20$, and $30 \mathrm{kHz}$. The contour plots cover a planar area measuring 4 feet on edge at a distance of 4 feet from a simulated point source, matching the mounting configuration shown in Figure 2. Note that the response contour features for the different frequencies are almost identical with a linear scaling factor being inversely proportional to frequency. The contour features would be more nearly identical if the array size were vanishingly small compared to the planar measuring area. However, given its 17 -inch radius, the array encompasses 39 degrees of solid collection angle at this distance. Included in Figure 4 are a series of line plots obtained by scanning through the contour plots in the $x_{0}$ direction for each $y_{\circ}$ location and selecting the maximum $\mathrm{dB}$ level. It can be seen that a plateau-like sidelobe structure exists at all frequencies, with the minimum sidelobe height approaching $-6 \mathrm{~dB}$ at a frequency of $20 \mathrm{kHz}$. 
A study of the beamwidth characteristics of the LADA can be achieved by observing a series of array responses for a number of frequencies spanning a range of $2-30 \mathrm{kHz}$ and measuring the width of the main lobe at various $\mathrm{dB}$ levels. Figure 5 shows a family of curves where the main lobe width is measured at the $-0.5,-1,-3$, and $-6 \mathrm{~dB}$ level. It can be seen from the curves that a typical $-3 \mathrm{~dB}$ beamwidth for the LADA is approximately 1.5 times the source wavelength.

Small Aperture Directional Array (SADA): The SADA is designed to complement the capabilities of the LADA by providing directivity and spectral information as a function of position around the model. The aperture of the array is kept small with the intent to keep all microphones in the array within approximately the same source directivity regardless of elevation or azimuth position. The array pattern which was chosen to achieve this can be seen in Figure 6, with the locations of the microphones given in Table 2 . The SADA consists of 33 B\&K model 4133, 1/8-inch microphones with $1 / 4$-inch preamplifiers projecting from an acoustically treated aluminum frame. The array pattern incorporates four irregular circles of eight microphones each with one microphone placed at $\vec{x}_{c}$, corresponding to the center of the array. Each circle is twice the diameter of the circle it encloses. The maximum radius of the array is 3.89 inches, giving the SADA only $5.25 \%$ of the surface area of the LADA. Two small laser diode pointers are incorporated into the array mount on opposite sides of the center microphone for use in alignment.

The SADA is mounted on a pivotal boom designed to allow it to be positioned to a wide range of elevation and azimuth angles while maintaining a constant distance to the center of the trailing edge of the main element airfoil (an assumed noise production region). This is achieved by maintaining the boom's pivot center at the trailing edge of the main element airfoil. Rotation of the boom is performed using precision DC servo rotation stages mounted on the outer edges of the side plates holding the model and boom. This is illustrated in Figure 7, which shows the SADA mounted in the QFF on the suction side of an airframe noise model at a 5-foot working distance. At this distance the array encompasses 7.5 degrees of solid collection angle.

Figure 8 shows a series of contour plots showing SADA array responses using equations (4) and (5) for $10,20,30$ and $40 \mathrm{kHz}$. Subsequently, a processing procedure is used to maintain constant spatial resolution, independent of frequency; however, this is not done in the calculations of Figure 8. The contour plots cover an area measuring 4 feet on edge at a distance of 5 feet from a simulated point source, matching the mounting configuration shown in Figure 7. A series of line plots obtained from the contour plots in a process similar to that for the LADA are also shown in Figure 8. It can be seen that the sidelobe patterns again exhibit a plateau-like structure at all frequencies, with the maximum sidelobe level approaching $-8 \mathrm{~dB}$ at a frequency of $40 \mathrm{kHz}$.

A study of the beamwidth characteristics of the SADA can be performed similarly to that for the LADA by observing a series of array responses over a frequency range and measuring the width of the main lobe at various $d B$ levels. Figure 9 shows such a beamwidth plot. A family of curves is shown where the main lobe width is measured at the $-0.5,-1,-3$, and $-6 \mathrm{~dB}$ level. It can be seen from these curves that a typical $3 \mathrm{~dB}$ beamwidth for the SADA is approximately 11 times the source wavelength. It will be seen subsequently that this beamwidth can be radically altered through the use of microphone shading (or weighting).

\section{Measurement System}

Data Acquisition: The data acquisition / analysis system employed for both arrays is illustrated in Figure 10. Acquisition hardware consists of a NEFF 495 transient data recorder which is controlled by a DEC AXP3400 workstation. Sampling rate is controlled by an external clock operating at $142.857 \mathrm{kHz}$. The maximum allowable clock rate is 1 MHz. The use of an external clock allows simultaneous acquisition with other instrumentation such as the model unsteady surface pressure sensors, as described in Reference 17. The NEFF system incorporates 36 12-bit (including sign bit) acquisition channels with each channel possessing a 4 megabyte buffer, allowing up to 2 million 2-byte samples to be collected per acquisition. The signals from each microphone channel are conditioned by passing them through high pass filters set to $300 \mathrm{~Hz}$ (to remove DC, $60 \mathrm{~Hz}$ line noise, and low frequency interference noise) and through anti-aliasing filters set at $50 \mathrm{kHz}$ which is substantially below the $71.43 \mathrm{kHz}$ Nyquist frequency.

Custom software is used to control all aspects of the data acquisition. The output files generated by the acquisition system are written in NetCDF format to provide platform-independent storage of the data, a feature mandated by the distributed data analysis system. ${ }^{20}$ The NetCDF files are archived on the NASA Langley Distributed Mass Storage Subsystem for posttest retrieval and processing. ${ }^{21}$ 
A typical acquisition run consists of collecting 36 channels (array microphones plus additional reference microphones) of data under no flow conditions. This is followed by the actual data run under a specific flow or calibration condition. As will be seen, spectra obtained from the background runs are subtracted from spectra obtained from data runs to remove the noise floor in the measurements.

Data Analysis: It was desired to build a highly distributed processing configuration to handle the problem of array analysis given the volume of data involved (greater than 500 Gbytes) and the amount of time required to process a single test point of data from start to finish (typically 30-60 minutes per set on a 200-MHz Pentium-Pro machine). There are a number of various platforms and operating systems used in the processing of the array data, including a cluster of three $200-\mathrm{MHz}$ NT-based Pentium-Pro workstations, a 500-MHz Alpha workstation running UNIX, and the Langley SP2 supercluster consisting of 48 IBM RS/6000 workstations. This heterogeneous cluster of hardware systems is controlled from a single PentiumPro workstation using a custom control panel program and a series of device independent configuration files readable by the individual processing codes located on each of the various hardware platforms.

\section{Data Post-Processing Procedure}

Processing steps common to both arrays include the construction of cross spectral matrices from the raw time data and the calculation of amplitude and time delay corrections to account for shear layer refraction. Classical beamformer processing algorithms are utilized in the generation of noise images, spectra, and directivity information. In addition, the SADA processing incorporates a unique shading algorithm which provides a constant beamwidth independent of frequency.

Computation of Cross Spectral Matrices: An $M$ by $M$ cross spectral matrix, where $M$ is the total number of microphones in the array, is first constructed for each data set (both background and airframe component test condition). The formation of the individual matrix elements is achieved through the use of Fast Fourier Transforms (FFT). This is done after converting the raw data to engineering units (Pascals) using sensitivity data based on a microphone calibration using a frequency of $1 \mathrm{kHz}$. Each channel of engineering unit data is then segmented into a series of non-overlapping blocks each containing 8192 samples, yielding a frequency resolution of $17.45 \mathrm{~Hz}$ for the $142.857 \mathrm{kHz}$ acquisition sampling rate. Using a Hamming window, each of these blocks of data is Fourier transformed into the frequency domain. The individual upper triangular matrix elements plus the diagonal (representing auto spectra for each array microphone) are formed by computing the corresponding block-averaged cross spectra from the frequency data using

$$
\hat{G}=\left[\begin{array}{cccc}
G_{11} & G_{12} & \cdots & G_{1 M} \\
& G_{22} & & \vdots \\
& & \ddots & \vdots \\
& & & G_{M M}
\end{array}\right]
$$

with

$$
G_{i j}(f)=\frac{1}{N W_{s}} \sum_{k=1}^{N}\left[X_{i k}^{*}(f) X_{j k}(f)\right]
$$

where $W_{s}$ is the data window weighting constant, $N$ is the number of blocks of data, and $X$ represents an FFT data block. The lower triangular elements of the matrix are formed by taking the complex conjugates of the upper triangular elements (allowed because the cross spectral matrix is Hermitian).

All cross spectral matrix elements are employed in subsequent processing, with no modification of the diagonal terms. Note that for in-flow arrays, the diagonal terms can be removed to improve the spectral dynamic range by subtracting off self-noise dominated auto-spectra during the beamforming process, as described in References 14 through 16 . However, for the airframe noise measurements described here, this step was not required since all array microphones are outside of the flow.

3-D Shear Layer Refraction Correction: Testing in an open-jet facility requires that the effect of the shear layer on the propagation of the noise (both intensity and retarded time) from sources located in the jet to microphones located outside the jet be accounted for. The first challenge was to develop a technique for dealing with the highly three dimensional, curved shear layer present in the installation. The approach taken was to acquire five-hole pitot probe measurements on both the pressure and suction sides of the airframe model to map out the velocity field. The shear layer position was defined to be the half mean velocity position. This data was then fitted with a three dimensional surface to provide a continuous representation of the shear layer for each of the flow conditions examined. With the shear layer position defined, amplitude and phase corrections were 
determined using the approach of Schlinker and Amiet $^{22}$ and Amiet ${ }^{23}$.

The key to finding the retarded time and phase corrections is to find the intersection of the source ray path with the shear layer, as illustrated in Figure 11. An iterative process is used, using the following relationship between the source emission angle, $\varphi_{1}$, the ray angle, $\theta$, and the free jet Mach number, $M_{0}$

$$
\tan (\theta)=\frac{\sin \left(\varphi_{1}\right)}{M_{0}+\cos \left(\varphi_{1}\right)}
$$

and Snell's law

$$
\cos \left(\varphi_{2}\right)=\frac{\cos \left(\varphi_{1}\right)}{1+M_{o} \cos \left(\varphi_{1}\right)}
$$

where the subscripts 1 and 2 refer to angles inside and outside of the jet, respectively, and the sound speeds inside and outside the jet are assumed equal. Once the ray path-shear layer intersection is known, the retarded time difference and hence the phase can be computed from

$$
\omega \Delta t=\omega\left(\frac{r_{\text {path }}-r_{\text {mic }}}{c_{0}}\right)
$$

where $r_{\text {path }}=r_{1}+r_{2}$ is the wavefront travel distance (relative to the convecting flow inside the jet), and $r_{\text {mic }}$ is the line-of-sight distance from the source to the microphone.

The amplitude corrections are based upon analysis of a rectangular shear layer. There are two corrections provided in Reference 22, namely a thick shear layer (high frequency) correction and a thin shear layer (low frequency) correction. The appropriate correction is determined by the ratio of the source acoustic wavelength to the shear layer thickness. The assumption in developing the thick shear layer correction is that the shear layer is sufficiently thick for geometrical acoustics to apply so that (1) the acoustic energy is conserved along the ray tube, and (2) sound pressure is the result of outgoing waves only since reflections are absent in the geometrical acoustics limit. As supplied in References 22 and 23, the ratio of the corrected to measured sound pressure for microphone $\mathrm{m}$, including the astigmatism and distance correction, is found to be

$$
A_{m}=\frac{p_{c}}{p_{m}}=\alpha_{1} \sqrt{\alpha_{2} \alpha_{3}}\left(1-M_{0} \cos \left(\varphi_{2}\right)\right) \sqrt{\frac{\xi\left(1+M_{\sigma}^{2} \xi^{2}\right)}{\sin \left(\varphi_{2}\right)}}
$$

with

$$
\begin{gathered}
\xi=\sqrt{\left(1-M_{o} \cos \left(\varphi_{2}\right)\right)^{2}-\cos ^{2}\left(\varphi_{2}\right)} \\
\alpha_{1}=\frac{\sin \theta_{\text {mic }}}{\sin \varphi_{2}} \\
\alpha_{2}=\frac{h}{r_{m i c} \sin \theta_{\text {mic }}}\left[\left(\frac{\sin \varphi_{2}}{\xi}\right)^{3}-1\right]+1 \\
\alpha_{3}=\frac{h}{r_{\text {mic }} \sin \theta_{\text {mic }}}\left[\left(\frac{\sin \varphi_{2}}{\xi}\right)-1\right]+1
\end{gathered}
$$

where $p_{c}$ is the corrected pressure, $p_{m}$ is the measured pressure, $h$ is the distance from source to shear layer, and $\theta_{m i c}$ is the measured angle of the microphone relative to the flow direction.

For the low frequency correction, the reflected wave amplitude cannot be neglected when the wavelength is of the same length as the shear layer thickness. In this case the amplitude correction is found to be

$$
\begin{aligned}
A_{m}=\frac{p_{c}}{p_{m}}= & \frac{1}{2} \alpha_{1} \sqrt{\alpha_{2} \alpha_{3}} \sqrt{1+M_{o}^{2} \xi^{2}} \times \\
& {\left[\frac{\xi}{\sin \left(\varphi_{2}\right)}+\left(1-M_{o} \cos \left(\varphi_{2}\right)\right)^{2}\right] }
\end{aligned}
$$

Examples of the calibration and use of the shear correction algorithms are shown subsequently.

Beamforming: A classical beamforming approach is used for the analysis which eliminates instabilities and potential matrix singularity problems found in adaptive techniques. The basic procedure consists of electronically steering the array to a predefined series of locations in space, as shown in Figure 12. These locations define a plane which can be positioned in any orientation in front of the array. For each selected steering location, a steering matrix containing one entry for each microphone in the array is computed as follows:

$$
\hat{\mathbf{e}}=\left[\begin{array}{c}
A_{1} \frac{r^{\circ}}{r_{m}^{\circ}} \exp \left\{-j\left[\left(\vec{k} \cdot \vec{x}_{1}\right)+\omega \Delta t_{1, \text { shear }}\right]\right\} \\
\vdots \\
A_{M} \frac{r^{\circ}}{r_{M}^{\circ}} \exp \left\{-j\left[\left(\vec{k} \cdot \vec{x}_{M}\right)+\omega \Delta t_{M, \text { shear }}\right]\right\}
\end{array}\right]
$$

where $\vec{x}$ is the distance from the steering location to each microphone, $A_{m}$ is the shear layer amplitude correction for microphone $m$ using either equation (10) 
or (12), and $\omega \Delta t_{m, s h a r}$ is the shear layer phase correction for microphone $m$ at frequency $\omega$. The factor $\left(r^{0} / r_{m}\right)$ is included to normalize the amplitude $A_{m}$ to that of the array $\vec{x}_{c}$ position. Using equation (13) and the cross spectral matrix computed previously, the steered array output power spectrum at the steering location is obtained via

$$
P(\hat{e})=\frac{\hat{\mathrm{e}}^{T}\left(\hat{G}_{\text {data }}-\hat{G}_{\text {badkround }}\right) \hat{\mathrm{e}}}{M}
$$

where the $T$ denotes the conjugate transpose of the matrix. Note that a background subtraction process is explicitly denoted in equation (14). The background spectra is that obtained without tunnel flow, where the acquisition system noise dominates the recorded output. The division by the number of microphones $M$ serves to reference the array output spectrum levels to equivalent single microphone output levels. Equation (14) represents the steered response power spectrum over the full range of single narrowband frequencies. If a wider bandwidth is desired (such as an Octave Band), the power (pressure-squared values) of the narrowbands is summed. Note that wider bandwidths are not formed prior to the completion of the vectorial (or complex) operations of equation (14). This prevents possible significant bias errors in summing across phase-shifted cross spectral bands.

SADA Shading Algorithm: The use of the SADA for directivity and spectral measurements requires that the beamwidth be invariant under steering angle and frequency changes, thereby providing a constant sensing area over noise source regions. The method used to accomplish this is similar to previous techniques described in Reference 12 and 13. The SADA microphones are divided into three clusters containing 17 microphones each. These clusters along with their maximum diagonal aperture sizes are shown in Table 3. Each cluster exhibits the same directional characteristics for a given wavenumber-length product $k D_{m}$, where $k$ is the wavenumber and $D_{n}$ is the diagonal distance between the elements of the n-th cluster. The method used to achieve the invariant sensing area consists of shading (or weighting) the array clusters as a function of frequency. The microphone cluster shadings are calculated as follows:

$$
\left.\begin{array}{l}
w_{1}=0 \\
w_{2}=0 \\
w_{3}=1
\end{array}\right\} \sigma_{1} \leq 0 \text { and } \sigma_{2} \leq 0
$$

$$
\left.\begin{array}{l}
\left.\begin{array}{l}
w_{1}=\sigma_{1}^{0.875} \\
w_{2}=1-\sigma_{1}^{0.875} \\
w_{3}=0 \\
w_{1}=0 \\
w_{2}=\sigma_{2}^{0.875} \\
w_{3}=1-\sigma_{2}^{0.875}
\end{array}\right\} 0<\sigma_{1} \leq 1 \\
w_{1}=1 \\
w_{2}=0 \\
w_{3}=0
\end{array}\right\} \sigma_{2} \leq 1
$$

with the shading coefficients defined by

$$
\begin{gathered}
\sigma_{1}=\frac{k D_{2}-k D_{0}}{k D_{2}-k D_{1}} \\
\sigma_{2}=\frac{k D_{3}-k D_{0}}{k D_{3}-k D_{2}}
\end{gathered}
$$

The value of $k D_{0}$ for this study is 36.38 , corresponding to frequencies of 10,20 , and $40 \mathrm{kHz}$ for clusters 3,2 , and 1, respectively (assuming a speed of sound of $1126 \mathrm{ft} / \mathrm{sec}$ ). This causes the SADA to yield the same effective resolution for all frequencies between 10 and $40 \mathrm{kHz}$, with smooth blending among frequencies. The exponent of the coefficients, 0.875 , was found to differ slightly from the array of References 12 and 13.

Figure 13 illustrates modified theoretical array responses for the SADA for frequencies of 10, 12.5, 15 and $17.5 \mathrm{kHz}$, using equations (4) and (5) with the shadings of equation (15) substituted for the $w_{m}$ term. Comparing the responses with those shown in Figure 8, note that the responses for 10,20 , and $40 \mathrm{kHz}$ are now identical, as are the ones for 12.5 and 25,15 and 30 , and 17.5 and $35 \mathrm{kHz}$. This clearly illustrates the frequency-invariant main and side lobe structure now exhibited by the array. Figure 14 shows a beamwidth plot for the shaded array. At higher frequencies the beamwidth, while invariant, now takes on the value exhibited at the $k D_{3}$ wavenumber-length product. In a sense the higher frequency beamwidths have been sacrificed to achieve frequency invariance. This is an acceptable trade-off; however, since accurate source directivity data can only be obtained over a broad frequency range if the sensing area of the array is held constant.

To extract noise spectra and directivity from data obtained with the SADA, the classical beamforming technique is employed with minor variations. First, a single steering location is chosen for the array, which is itself positioned at various elevation and azimuth 
angles with respect to the model. A modified version of equation (14) is used to compute the weighted steered response power for the array at the fixed steering location via

$$
P(\hat{e})=\frac{\hat{\mathrm{e}}^{T} \hat{W}\left(\hat{G}_{\text {data }}-\hat{G}_{\text {background }}\right) \hat{W}^{T} \hat{\mathbf{e}}}{\sum_{m=1}^{M} w_{m}}
$$

where $\hat{W}$ is a row matrix containing the set of shadings computed in equation (15). The sum over the microphone shading terms in the denominator is obtained from equation (15) as a function of frequency (this sum always equals 17 for the present SADA application). Note that this formulation of the beamformer equation is identical to that for the LADA if one assumes an identity matrix for $\hat{W}$.

\section{Array Calibration and Applications}

Careful calibrations are conducted for both array systems. These tests are designed to check for deviations between experimental and theoretical array responses which can be attributed to microphone system response differences, installation effects, or problems in the data analysis algorithms.

Injection Calibration: Injection calibrations are performed for the SADA. These calibrations consist of inserting a known signal simultaneously into all microphone channels in order to detect microphone sensitivity and phase drift. Both pure tones and white noise are used. This is accomplished without physical disruption of the system. Inspection of cross spectral phase between all pairs of microphones allows discrepancies to be easily identified and corrected. Also, sensitivity drift can be corrected without the need to perform a full microphone calibration before each run. Nevertheless, standard SADA microphone calibrations are also performed daily because of ready accessibility.

Isolated Point Source: A series of static calibration tests are performed by placing an isolated point source directly in front of the array at the operational working distance ( 4 feet for the LADA, 5 feet for the SADA). The point source consists of a tube with one or more acoustic drivers mounted on the back end. The open end of the tube is intended to provide an omnidirectional sound source. Noise measurements are obtained across a broad frequency band though the use of white noise. These are compared with corresponding theoretical array responses using equations (4) and (5).

Figure 15 shows a series of LADA point source noise images taken at identical frequencies to the theoretical ones shown in Figure 4. Figure 16 shows the corresponding measured beamwidths which can be compared with Figure 5. At the higher frequencies some discrepancies were indicated between the theoretical and experimental sidelobe shapes (most likely due to installation effects); however, the measured beamwidths and peak sidelobe levels agree well with theory. Figures 17 and 18 show a series of SADA noise images and beamwidth line plots corresponding to the blended theoretical ones shown in Figures 13 and 14 , respectively. The shading algorithm is seen to be validated.

In-Situ Point Source: In addition to the static calibrations using an isolated point source, tests are conducted using the point source mounted in the QFF at the midpoint of the trailing edge of the main element airfoil. These measurements include:

- Background acquisition runs for no tunnel airflow. The corresponding spectra is subtracted from the other spectra to remove the noise floor, as described previously.

- Acquisition runs with no flow and point source turned on to verify processing accuracy and the effect of the test apparatus on the acoustic field.

- Acquisition runs with flow and point source operating to evaluate the shear layer correction algorithms.

Figure 19 shows a series of noise image maps taken with the SADA on the pressure side of the model for an elevation angle of 107 degrees and an azimuth angle of zero degrees. At this location the face of the array is parallel to the chord of the main element airfoil. Figure 19(a) shows a photograph of the point source mounted in the QFF pointing toward the pressure side of the model. Figure 19(b) shows a $30-\mathrm{kHz}$ noise image map of the point source under no flow conditions. Figure $19(\mathrm{c})$ shows a $30-\mathrm{kHz}$ noise image map for the point source operating in a Mach 0.17 flow with no shear layer correction applied, while Figure 19(d) shows a similar map with corrections. Notice that the apparent location of the source moves approximately 3 inches downstream of its actual position without shear layer corrections applied. The shear layer algorithm returns the source to its proper position, as verified by the no flow case. Other SADA elevation angles, producing larger 
position corrections, also find success using these algorithms.

\section{Test Application}

LADA Measurements: Acoustic noise image maps are obtained by steering the LADA over a plane parallel to the main element chord on the model pressure side. Because of limitations in the data acquisition process in the early test stages of the program (of which this particular LADA data was obtained), background noise spectra have not been subtracted. However, the effect of background noise was determined to be negligible for these results. Figure 20 shows typical acoustic image maps taken at frequencies of $5,8,12.5$, and $20 \mathrm{kHz}$. The flow is from bottom to top and an outline of the wing and flap provide a reference for the noise sources that are predominant. Note that the location and strength of the sources are dependent on frequency, with the level diminishing with frequency.

The benefits of using a larger aperture with corresponding narrow beamwidth can be seen in Figure 21. This figure shows the position of the locally dominant noise source location, defined by the centroid of the source on the image maps in Figure 20. This figure shows that along the flap-side edge, a trend exists for the lower frequency sound sources to be located near the flap trailing edge with the source location moving to the flap mid chord and flap main element juncture at higher frequencies. Such information is only obtainable using an array with a sufficiently large aperture size and correspondingly narrow beamwidth.

SADA Measurements: Figure 22 shows the SADA elevation angles which were employed for directivity studies in the QFF. Figure 23 shows flap edge spectra taken at an elevation angle of 107 degrees. The SADA azimuth angle is at zero degrees, corresponding to the plane of the flap side edge surface. The model flap angle condition is 29 degrees. The array is focused on the flap region, which for this flap angle is by far the most intense noise producing region. Shown on the plot, along with the SADA beamformed-output spectrum, is the spectra obtained from a single microphone in the array. The difference in levels between these represents the removal of unwanted noise emanating from regions other than those present at the steering location. As previously indicated, the SADA spectrum represents that noise emitted from a region of constant size for frequencies between 10 and $40 \mathrm{kHz}$. At lower frequencies, the noise emission region measured is larger; for higher frequencies, the region is smaller.

Figure 24 shows the elevation angle source directivity in terms of a series of noise spectra obtained for the SADA at a number of elevation angles about the model. The model flap angle is now 39 degrees. With the exception of the most downstream position, the spectra are within 2 to $3 \mathrm{~dB}$ of one another for frequencies from $10-30 \mathrm{kHz}$. Larger deviations in directivity occur over the lower and upper frequencies due to differences in source characteristics, as described in Reference 17.

It is noted that the LADA's 39 degrees of solid collection angle sets well within the SADA elevation angle range shown here. The degree of directivity uniformity found over the 10-30 kHz range of frequencies suggests that measurements with the LADA should have quantitative accuracy, in addition to it having source positioning accuracy. This is true, as long as the azimuthal directivity (not determined for this paper) is likewise uniform and that the spatial source-noise coherence is high. As previously indicated, any lack of spatial uniformity over the array microphones would effectively shade the microphone's response in the beamforming and, thus, would change the array response characteristics.

\section{Summary}

This paper presents an overview of the design and construction of two complementary microphone directional arrays used for aeroacoustic testing. A Large Aperture Directional Array (LADA) has been constructed to obtain high resolution noise localization maps. A Small Aperture Directional Array (SADA) has also been made to be moved about the model to provide localized spectra and directivity from selected noise source regions. Calibration tests have demonstrated their accuracy and functionality. Both arrays have been used to successfully measure the far field acoustics on a main element / half-span flap model. The LADA was able to detect small changes in location of dominant noise sources emanating from the flap edge region, while the SADA was able to obtain spectra and directivity measurements from this region.

\section{Acknowledements}

The authors wish to acknowledge Dave Devilbiss of Lockheed-Martin and Stuart Pope of Computer Sciences Corporation for data processing and software development support. The authors also gratefully acknowledge Phil Grauberger and Ron Verhapen of Wyle Laboratories for data acquisition system support. 


\section{References}

1. Kendall, J.M., Jr., "Airframe Noise Measurements by Acoustic Imaging", ALAA Paper 77-55, ALAA $15^{\text {th }}$ Aerospace Sciences Meeting, Los Angeles, CA, January, 1977.

2. Grosche, F.R, Stiewitt, H., and Binder, B., "Acoustic Wind-Tunnel Measurements with a Highly Directional Microphone", ALAA Journal, Volume 15, Number 11, pp. 1590-1596, 1977.

3. Schlinker, R.H., "Airfoil Trailing Edge Noise Measurements With a Directional Microphone", ALAA Paper 77-1269, $4^{\text {th }}$ ALAA Aeroacoustics Conference, Atlanta, GA, October, 1977.

4. Grosche, F.R, Schneider, G., and Stiewitt, H., "Wind Tunnel Experiments on Airframe Noise Sources of Transport Aircraft", ALAA Paper 971642, $3^{\text {rd }}$ ALAA/CEAS Aeroacoustics Conference, Atlanta, GA, May, 1997.

5. Brooks, T.F., Marcolini, M.A., Pope, D.S., "Airfoil Trailing-Edge Flow Measurements", ALAA Journal, Volume 24, Number 8, pp. 1245$1251,1986$.

6. Brooks, T.F., Pope, D.S., Marcolini, M.A., "Airfoil Self-Noise and Prediction", NASA Reference Publication 1218, July, 1989.

7. Elliot, R.S., "The Theory of Antenna Arrays", Microwave Scanning Antennas, R.C. Hansen, ed., Academic Press, 1966.

8. Burdic, W.S., "Underwater Acoustic System Analysis", Prentice-Hall, Inc., Englewood Cliffs, NJ, 1984.

9. Soderman, P.T., and Noble, S.C., "A FourElement End-Fire Microphone Array for Acoustic Measurements in Wind Tunnels", NASA Technical Memorandum X-62, 331, January, 1974.

10. Soderman, P.T., and Noble, S.C., "Directional Microphone Array for Acoustic Studies of Wind Tunnel Models", Journal of Aircraft, pp. 169-173, 1975.

11. Billingsley, J., and Kinns, R, "The Acoustic Telescope", Journal of Sound and Vibration, Volume 48, Number 4, pp. 485-510, 1976.
12. Brooks, T.F., Marcolini, M.A., and Pope, D.S., “A Directional Array Approach for the Measurement of Rotor Noise Source Distributions with Controlled Spatial Resolution", Journal of Sound and Vibration, Volume 112, Number 1, pp. 192197, 1987.

13. Marcolini, M.A., and Brooks, T.F., "Rotor Noise Measurement Using a Directional Microphone Array", Journal of the American Helicopter Society, pp. 11-22, 1992.

14. Underbrink, J.R., "Practical Considerations in Focused Array Design for Passive Broadband Source Mapping Applications", Master's Thesis, The Pennsylvania State University, May, 1995.

15. Mosher, M., "Phased Arrays for Aeroacoustic Testing: Theoretical Development", ALAA Paper 96-1713, $2^{\text {nd }}$ AIAA/CEAS Aeroacoustics Conference, State College, PA, May, 1996.

16. Watts, M.E., Mosher, M., and Barnes, M.J., "The Microphone Array Phased Processing System (MAPPS)", ALAA Paper 96-1714, $2^{\text {nd }}$ ALAA/CEAS Aeroacoustics Conference, State College, PA, May, 1996.

17. Meadows, K.R., Brooks, T.F., Humphreys, W.M., Hunter, W.W., and Gerhold, C.H., "Aeroacoustic Measurements of a Wing-Flap Configuration", AIAA Paper 97-1595, $3^{\text {rd }}$ ALAA/CEAS Aeroacoustics Conference, Atlanta, GA, May, 1997.

18. Johnson, D.H., and Dudgeon, D.E., Array Signal Processing, Prentice Hall, 1993.

19. Hubbard, H.H., and Manning, J.C., Aeroacoustic Research Facilities at NASA Langley Research Center, NASA Technical Memorandum 84585, 1983.

20. Rew, R., Davis, G., Emmerson, S., and Davies, H., "NetCDF User's Guide - An Interface for Data Access", University Corporation for Atmospheric Research - Unidata Program Center, Boulder, CO, 1996.

21. Pao, J.Z., and Humes, D.C., "NASA Langley Research Center's Distributed Mass Storage System", $14^{\text {th }}$ IEEE Symposium on Mass Storage Systems, 1995. 
22. Schlinker, RH., and Amiet, RK., "Shear Layer Refraction and Scattering of Sound", AlAA Paper 80-973, 1980.
23. Amiet, R.K., "Refraction of Sound by a Shear Layer", Journal of Sound and Vibration, Volume 58, Number 3, pp. 467-482, 1978.

Table 1 - LADA Microphone Coordinates (Viewed from Front of Array)

\begin{tabular}{|c|c|c|c|c|c|c|c|}
\hline Mic \# & X location & $Y$ location & Z location & Mic * & X location & $Y$ location & $Z$ location \\
\hline & (in) & (in) & (in) & & (in) & (in) & (in) \\
\hline 1 & 0.03 & -1.02 & 0.00 & 19 & -9.99 & 5.66 & 0.00 \\
\hline 2 & 0.97 & -0.32 & 0.00 & 20 & -8.47 & -7.78 & 0.00 \\
\hline 3 & 0.61 & 0.80 & 0.00 & 21 & 10.57 & -8.60 & 0.00 \\
\hline 4 & -0.57 & 0.81 & 0.00 & 22 & 11.44 & 7.37 & 0.00 \\
\hline 5 & -0.93 & -0.30 & 0.00 & 23 & -3.47 & 13.14 & 0.00 \\
\hline 6 & -5.13 & 0.88 & 0.00 & 24 & -13.56 & 0.75 & 0.00 \\
\hline 7 & -2.42 & -4.63 & 0.00 & 25 & -4.89 & -12.69 & 0.00 \\
\hline 8 & 3.67 & -3.75 & 0.00 & 26 & 14.55 & -5.15 & 0.00 \\
\hline 9 & 4.71 & 2.30 & 0.00 & 27 & 9.39 & 12.22 & 0.00 \\
\hline 10 & -0.73 & 5.17 & 0.00 & 28 & -8.73 & 12.68 & 0.00 \\
\hline 11 & -2.34 & -8.63 & 0.00 & 29 & -14.74 & -4.38 & 0.00 \\
\hline 12 & 7.50 & -4.91 & 0.00 & 30 & -0.39 & -15.42 & 0.00 \\
\hline 13 & 6.99 & 5.59 & 0.00 & 31 & 17.01 & -1.12 & 0.00 \\
\hline 14 & -3.16 & 8.35 & 0.00 & 32 & 6.32 & 15.81 & 0.00 \\
\hline 15 & -8.92 & -0.42 & 0.00 & 33 & -13.08 & 10.89 & 0.00 \\
\hline 16 & 4.80 & -10.49 & 0.00 & 34 & -14.39 & -9.09 & 0.00 \\
\hline 17 & 11.45 & 1.30 & 0.00 & 35 & 4.21 & -16.52 & 0.00 \\
\hline 18 & 2.31 & 11.28 & 0.00 & & & & \\
\hline
\end{tabular}

Table 2 - SADA Microphone Coordinates (Viewed from Front of Array)

\begin{tabular}{|c|c|c|c|c|c|c|c|}
\hline Mic \# & X location & $Y$ location & $Z$ location & Mic & X location & $Y$ location & $Z$ location \\
\hline & (in) & (in) & (in) & & (in) & (in) & (in) \\
\hline 1 & 0.00 & 0.00 & 0.00 & 18 & 0.00 & -1.80 & 0.00 \\
\hline 2 & 0.00 & -0.45 & 0.00 & 19 & 1.37 & -1.37 & 0.00 \\
\hline 3 & 0.34 & -0.34 & 0.00 & 20 & 1.80 & 0.00 & 0.00 \\
\hline 4 & 0.45 & 0.00 & 0.00 & 21 & 1.37 & 1.37 & 0.00 \\
\hline 5 & 0.34 & 0.34 & 0.00 & 22 & 0.00 & 1.80 & 0.00 \\
\hline 6 & 0.00 & 0.45 & 0.00 & 23 & -1.37 & 1.37 & 0.00 \\
\hline 7 & -0.34 & 0.34 & 0.00 & 24 & -1.80 & 0.00 & 0.00 \\
\hline 8 & -0.45 & 0.00 & 0.00 & 25 & -1.37 & -1.37 & 0.00 \\
\hline 9 & -0.34 & -0.34 & 0.00 & 26 & 0.00 & -3.60 & 0.00 \\
\hline 10 & 0.00 & -0.90 & 0.00 & 27 & 2.75 & -2.75 & 0.00 \\
\hline 11 & 0.69 & -0.69 & 0.00 & 28 & 3.60 & 0.00 & 0.00 \\
\hline 12 & 0.90 & 0.00 & 0.00 & 29 & 2.75 & 2.75 & 0.00 \\
\hline 13 & 0.69 & 0.69 & 0.00 & 30 & 0.00 & 3.60 & 0.00 \\
\hline 14 & 0.00 & 0.90 & 0.00 & 31 & -2.75 & 2.75 & 0.00 \\
\hline 15 & -0.69 & 0.69 & 0.00 & 32 & -3.60 & 0.00 & 0.00 \\
\hline 16 & -0.90 & 0.00 & 0.00 & 33 & 2.75 & -2.75 & 0.00 \\
\hline 17 & -0.69 & -0.69 & 0.00 & & & & \\
\hline
\end{tabular}

Table 3 - SADA Cluster Groupings

\begin{tabular}{|c|c|c|}
\hline Cluster Number & Microphone Grouping & Diagonal Cluster Aperture \\
\hline & & (in) \\
\hline 1 & $1-17$ & 1.94 \\
\hline 2 & $1,10-25$ & 3.88 \\
\hline 3 & $1,18-33$ & 7.76 \\
\hline
\end{tabular}




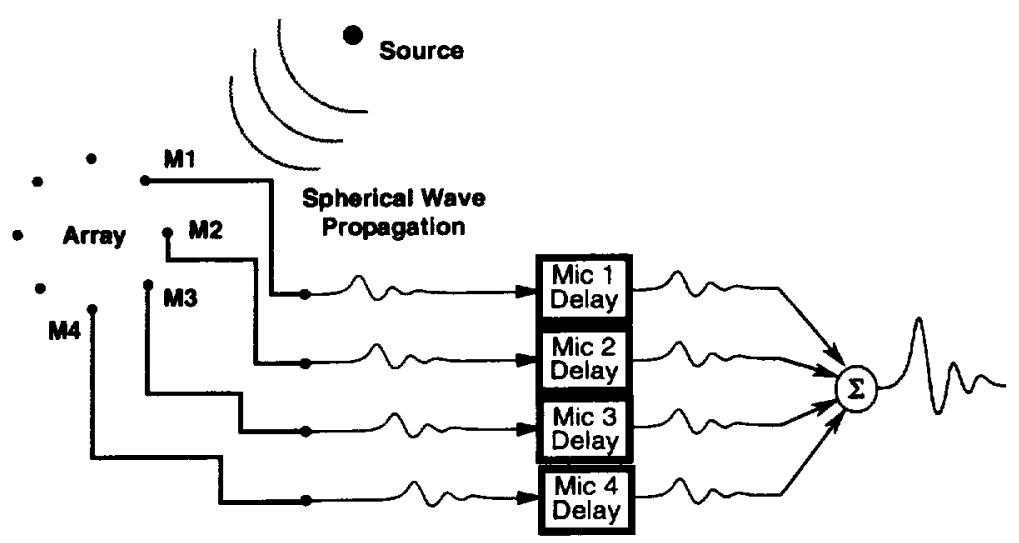

Figure 1. Basic Principle of Directional Array Operation.

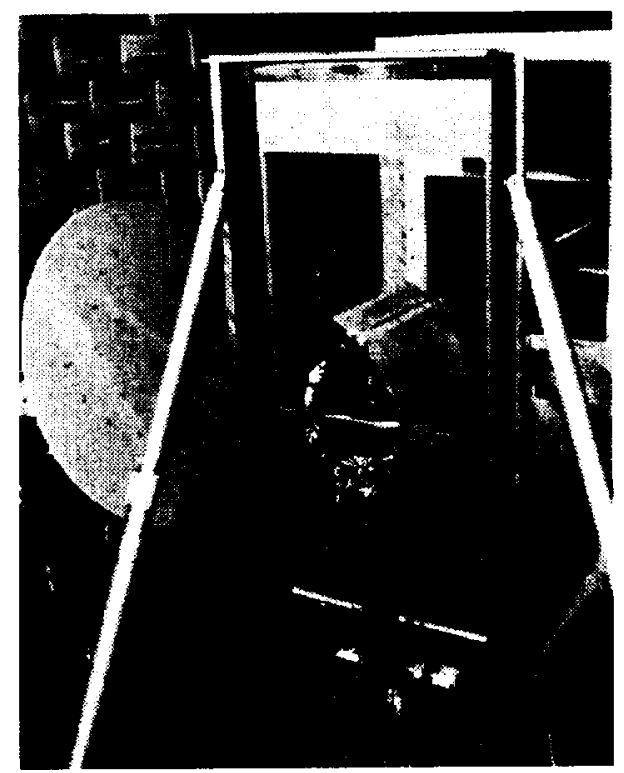

Figure 2. Large Aperture Directional Array Mounted in QFF for Testing.

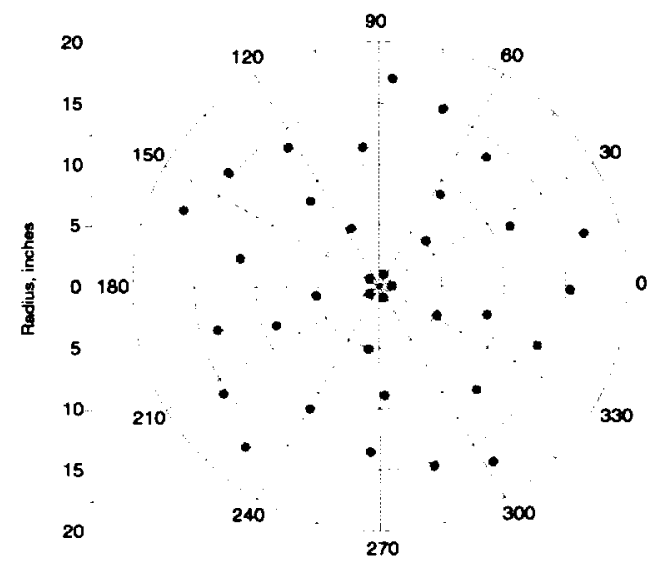

Figure 3. Large Aperture Directional Array Microphone Layout. 

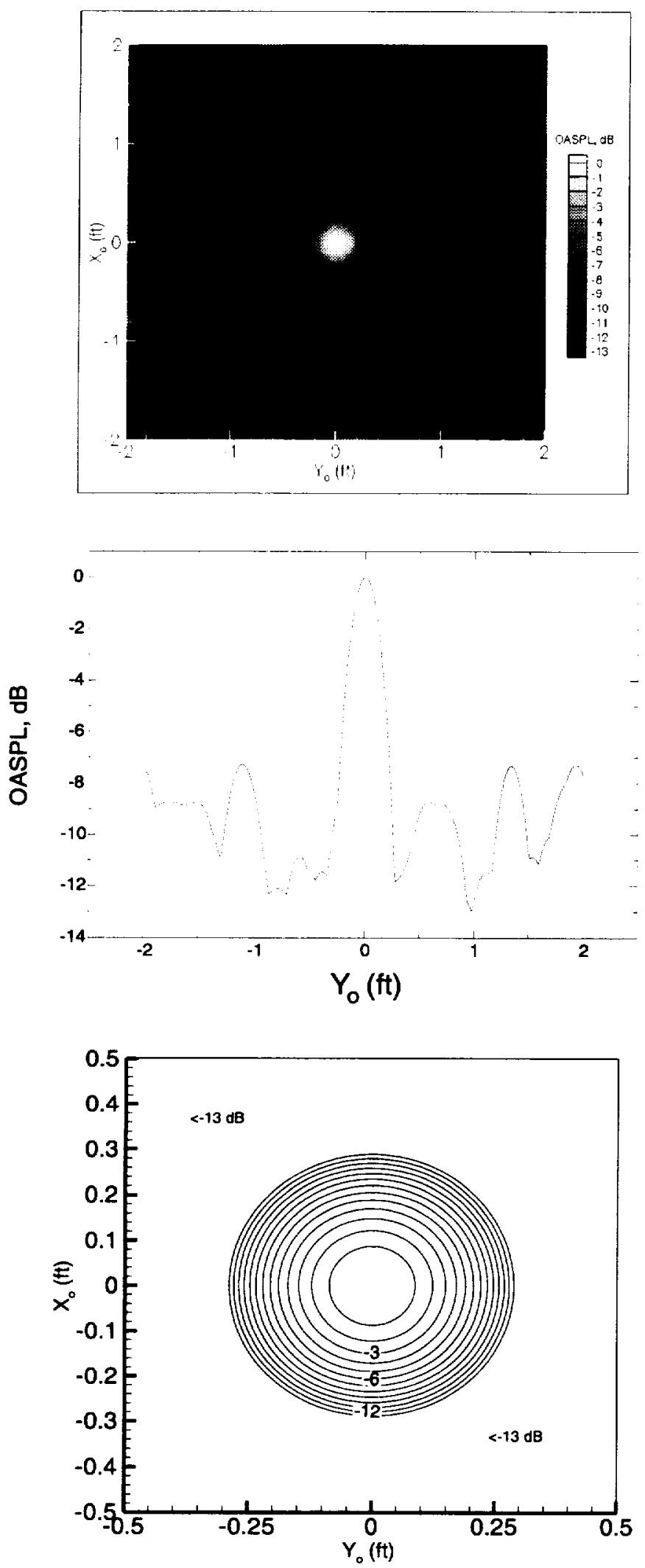

$6 \mathrm{kHz}$
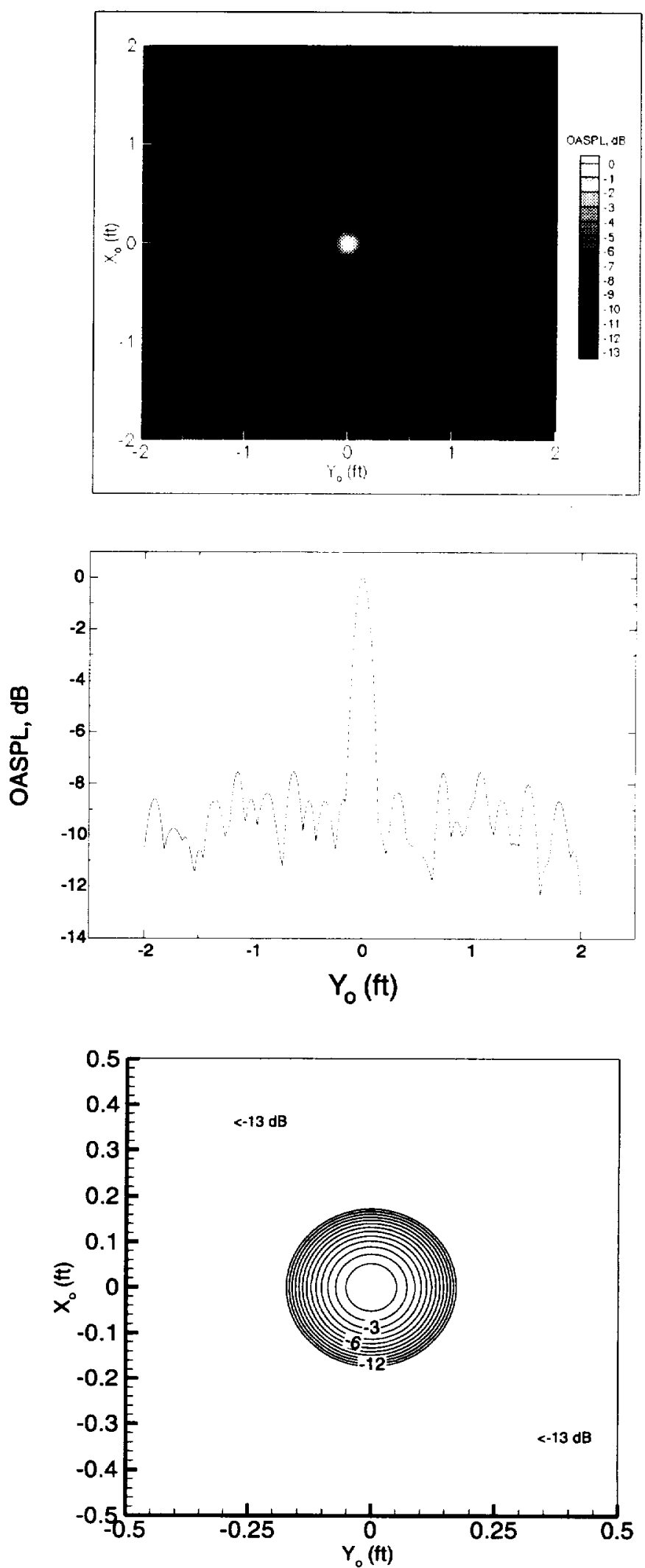

$10 \mathrm{kHz}$

Figure 4. LADA Theoretical Array Responses for $6,10,20$, and $30 \mathrm{kHz}$. 

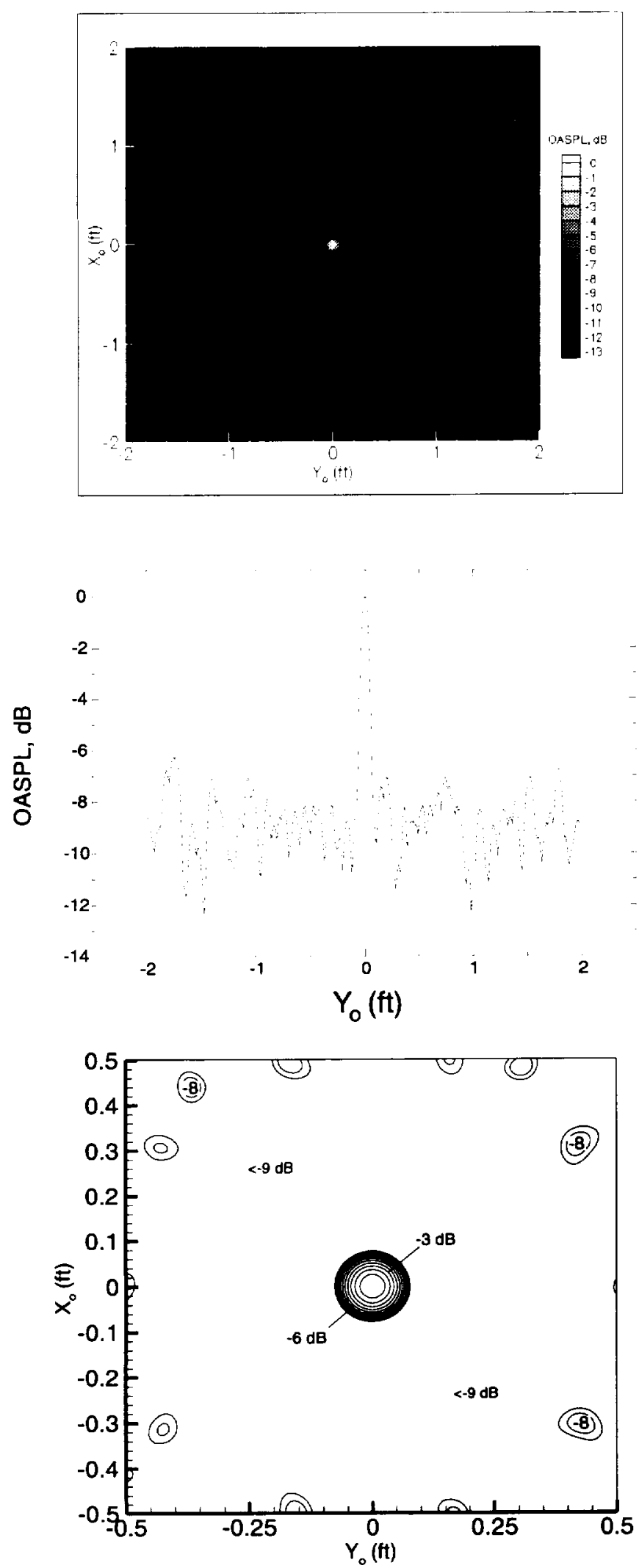

$20 \mathrm{kHz}$
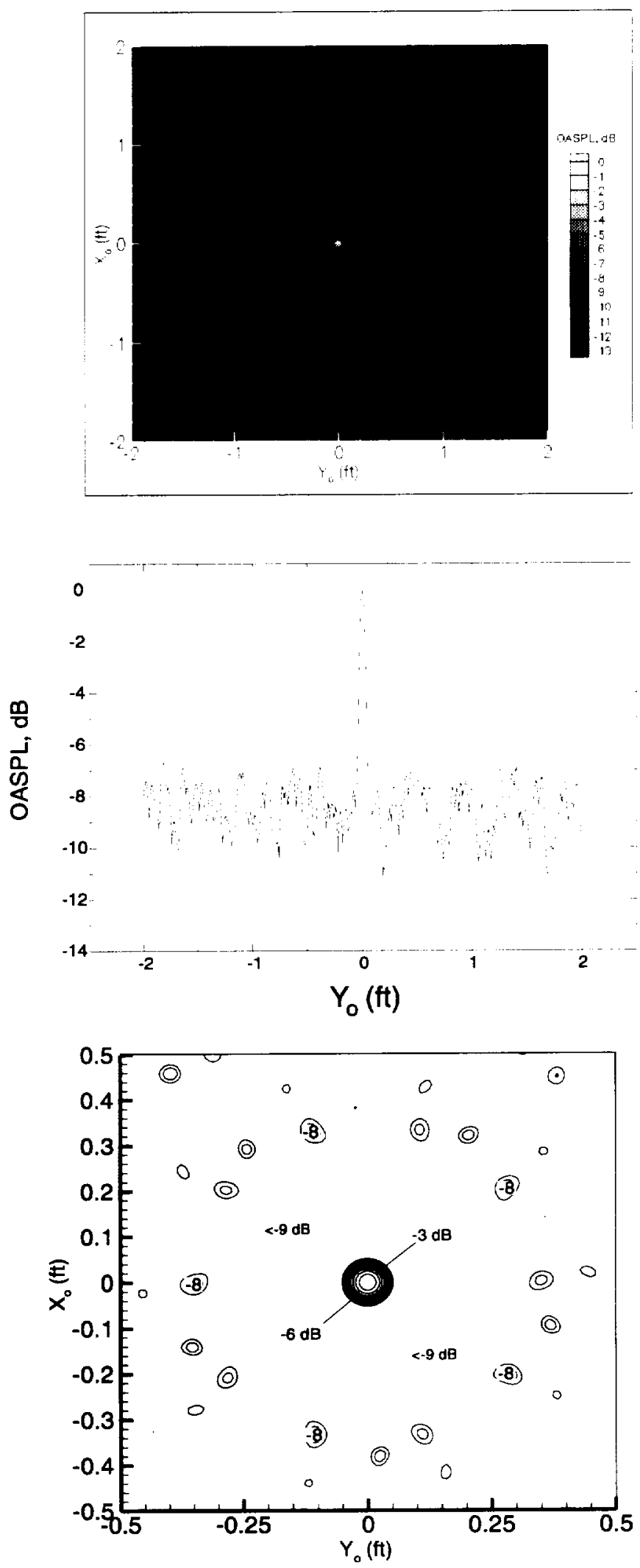

$30 \mathrm{kHz}$

Figure 4 (continued). LADA Theoretical Array Responses

for $6,10,20$, and $30 \mathrm{kHz}$. 


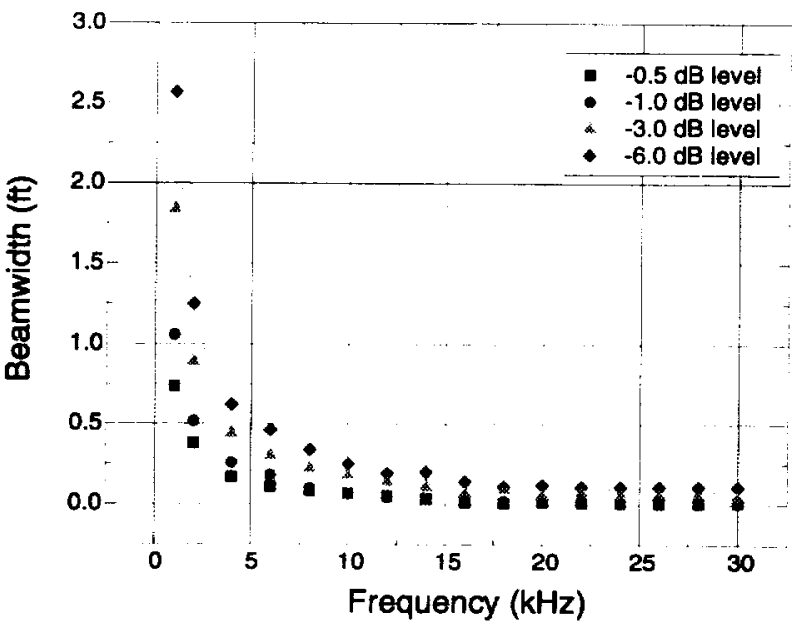

Figure 5. LADA Theoretical Beamwidth as Function of Frequency.

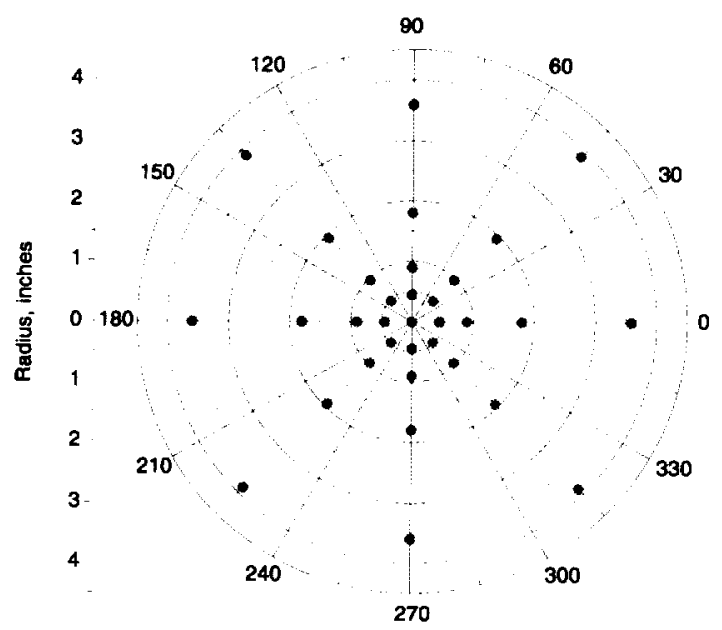

Figure 6. Small Aperture Directional Array Microphone Layout.

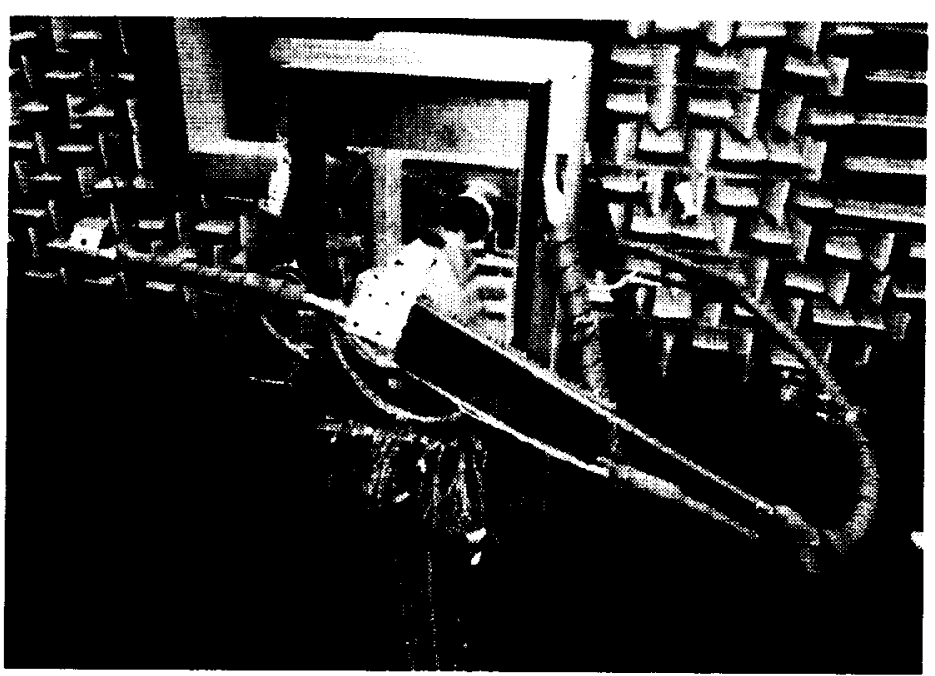

Figure 7. Small Aperture Directional Array Mounted in QFF for Testing.

American Institute of Aeronautics and Astronautics 

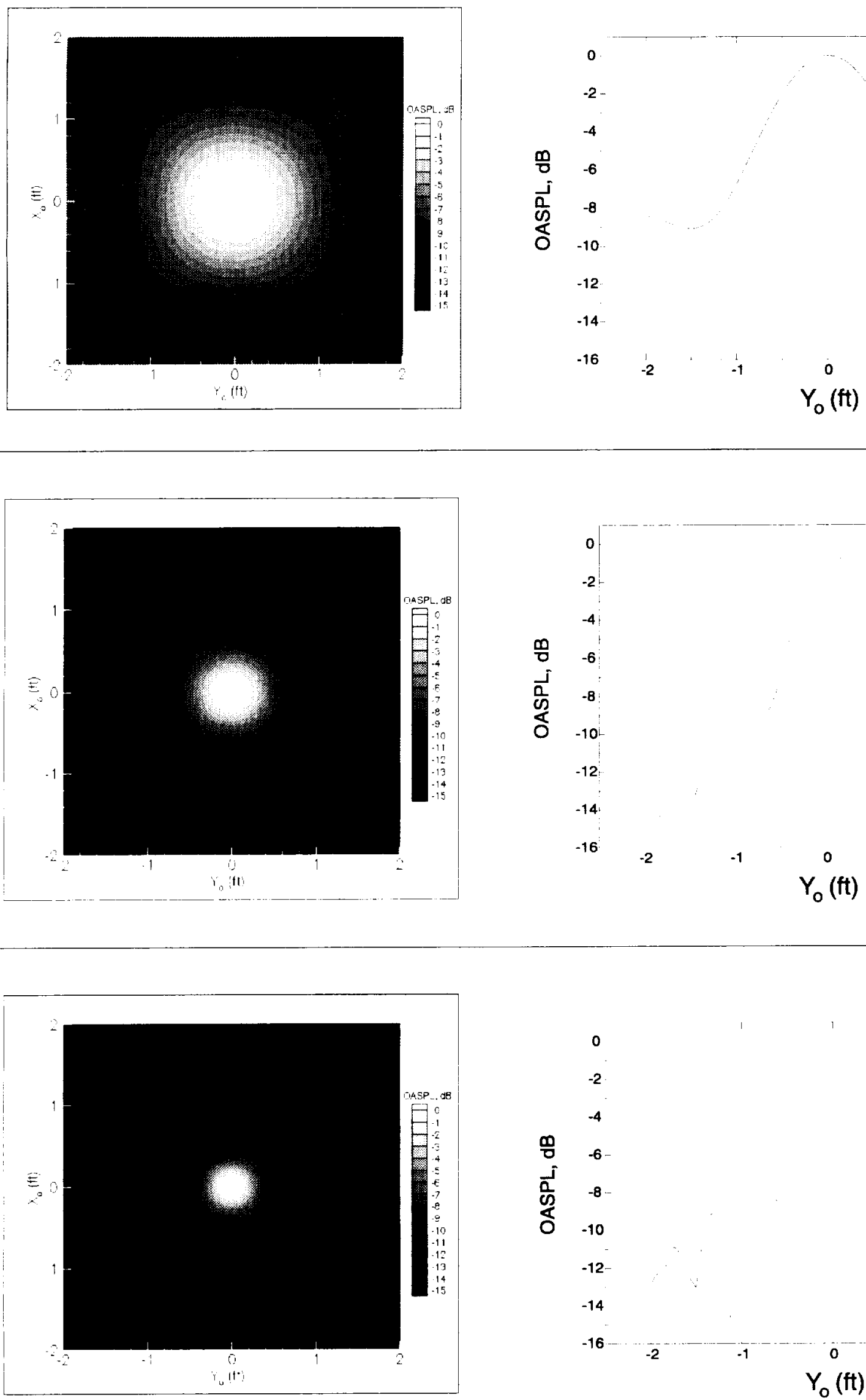

Figure 8. SADA Theoretical Array Responses for $10,20,30$, and $40 \mathrm{kHz}$. 

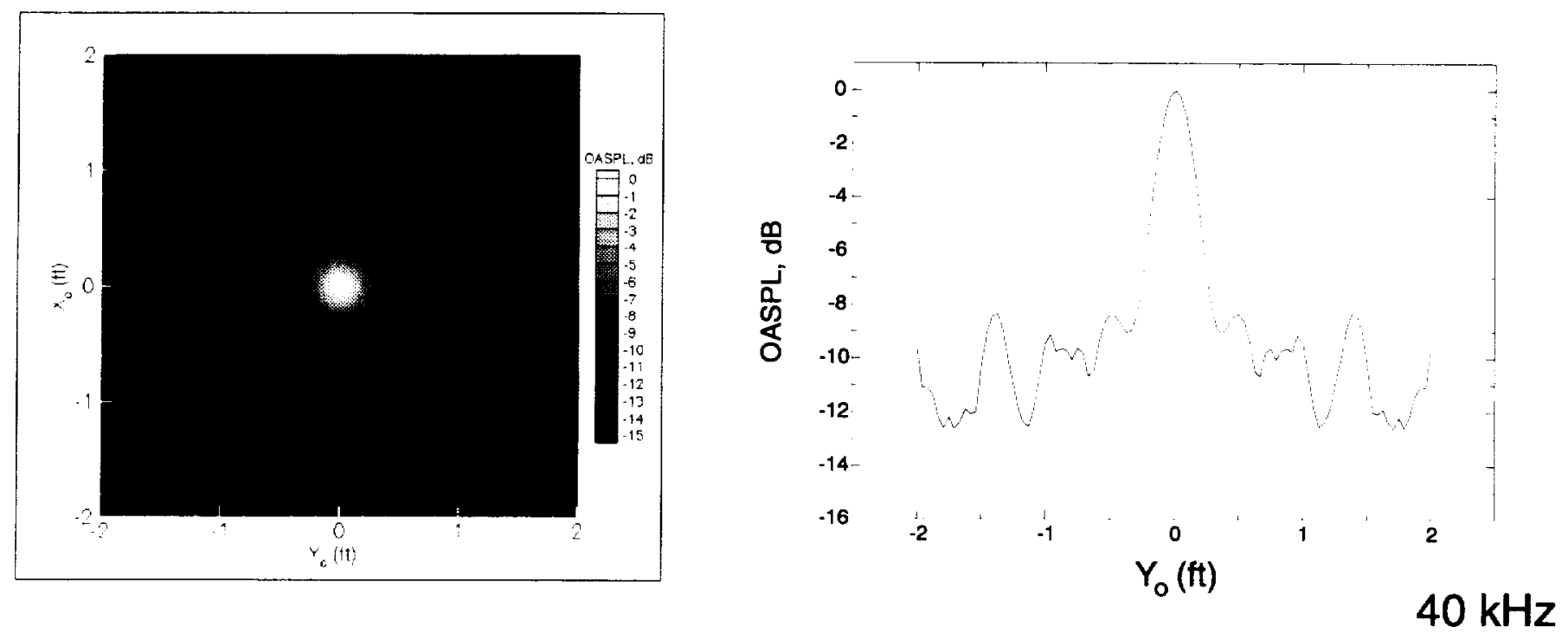

Figure 8 (continued). SADA Theoretical Array Responses for $10,20,30$, and $40 \mathrm{kHz}$

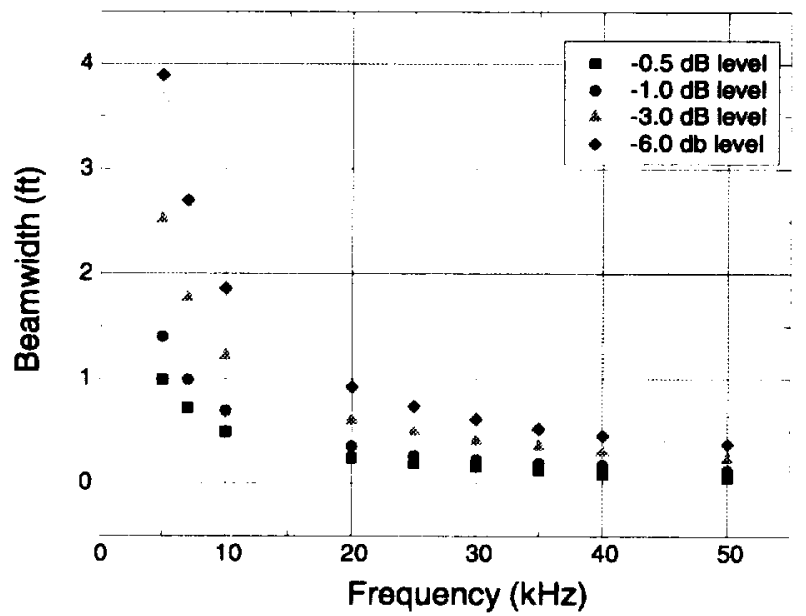

Figure 9. SADA Theoretical Beamwidth as Function of Frequency.

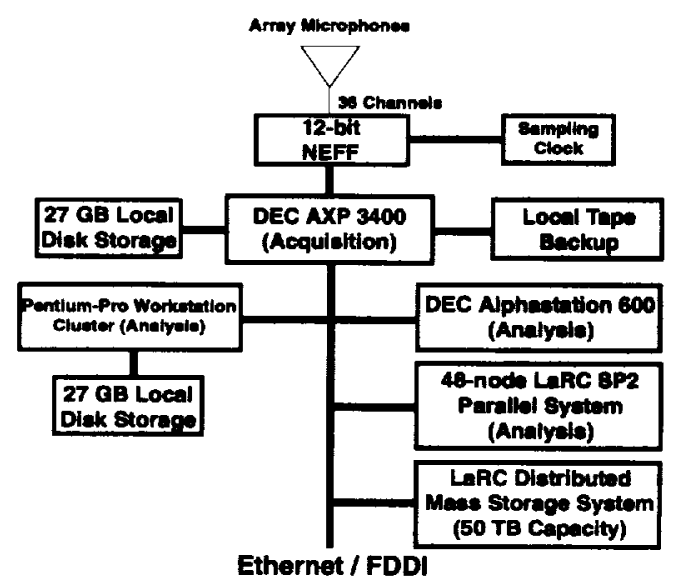

Figure 10. Data Acquisition / Analysis Block Diagram. 


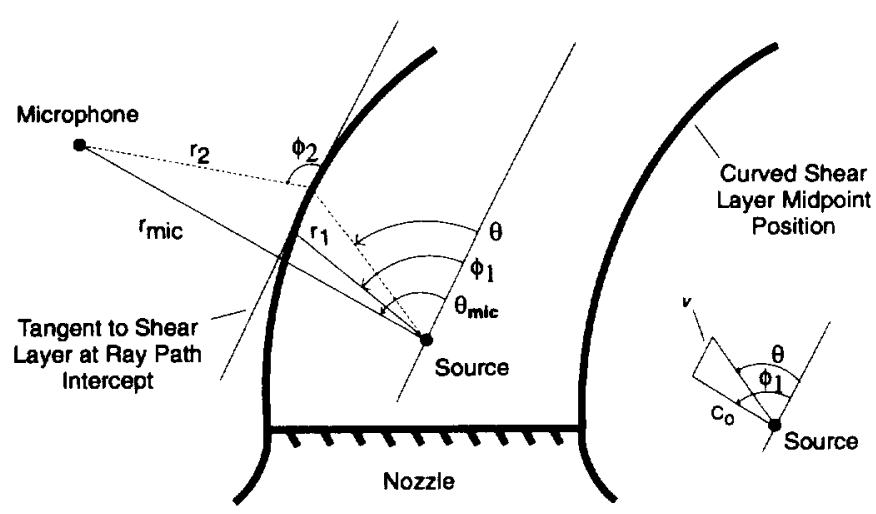

Figure 11. Geometry Showing Angles Utilized in Shear Layer Correction. Dashed Line Denotes Ray Path. Velocity Triangle is Shown.
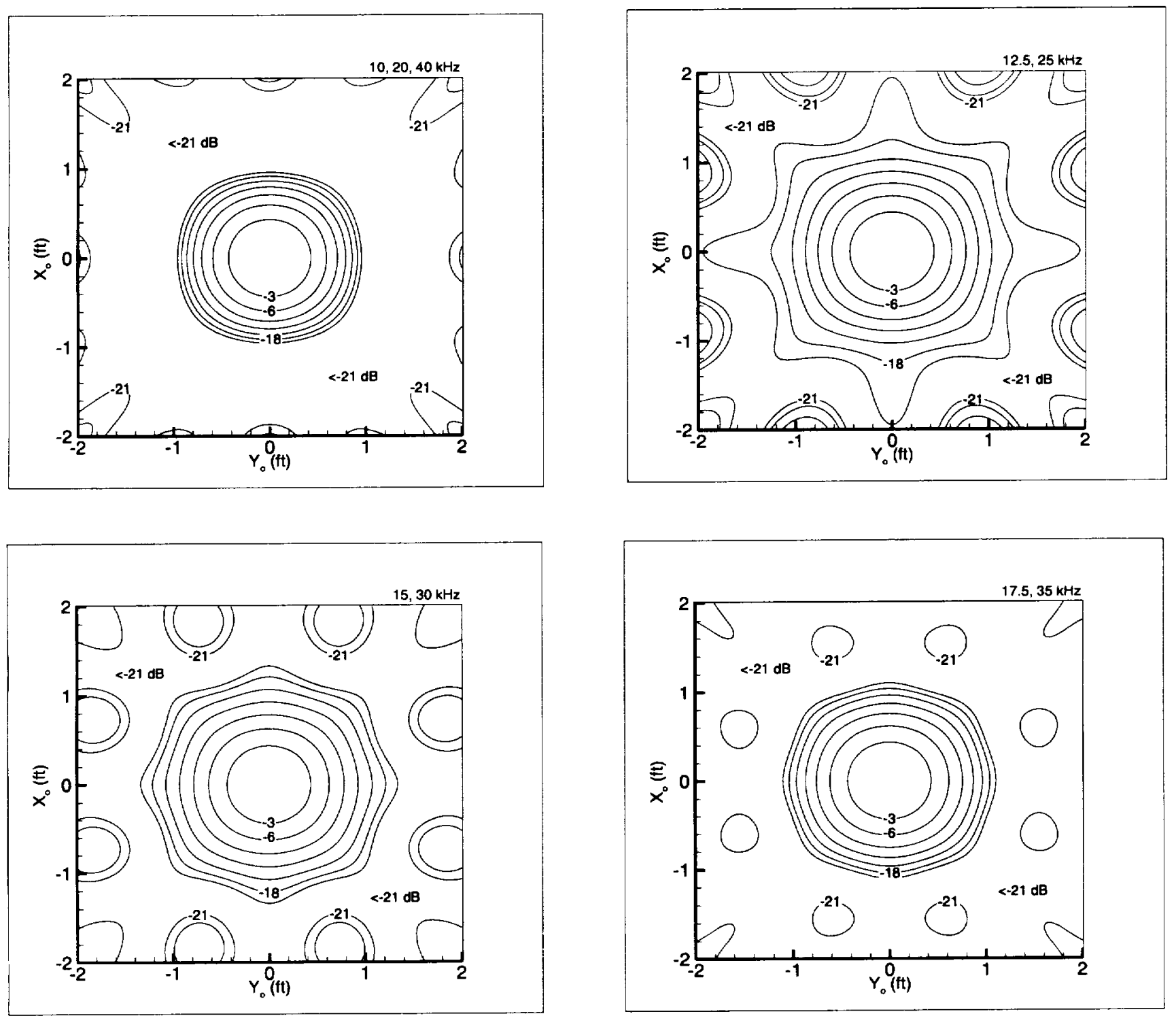

Figure 13. Frequency-Invariant SADA Theoretical Array Responses. 


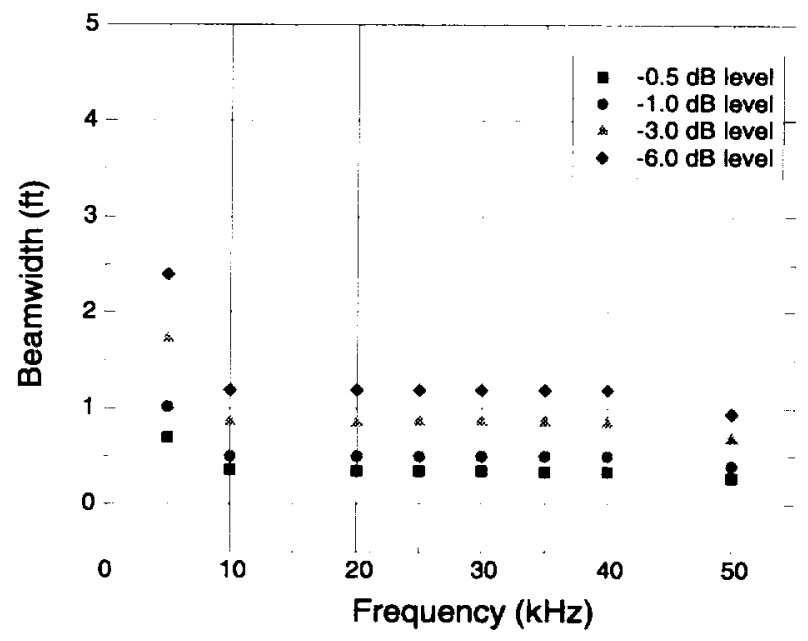

Figure 14. SADA Theoretical Beamwidths Using Shading.

Compare with Figure 9.
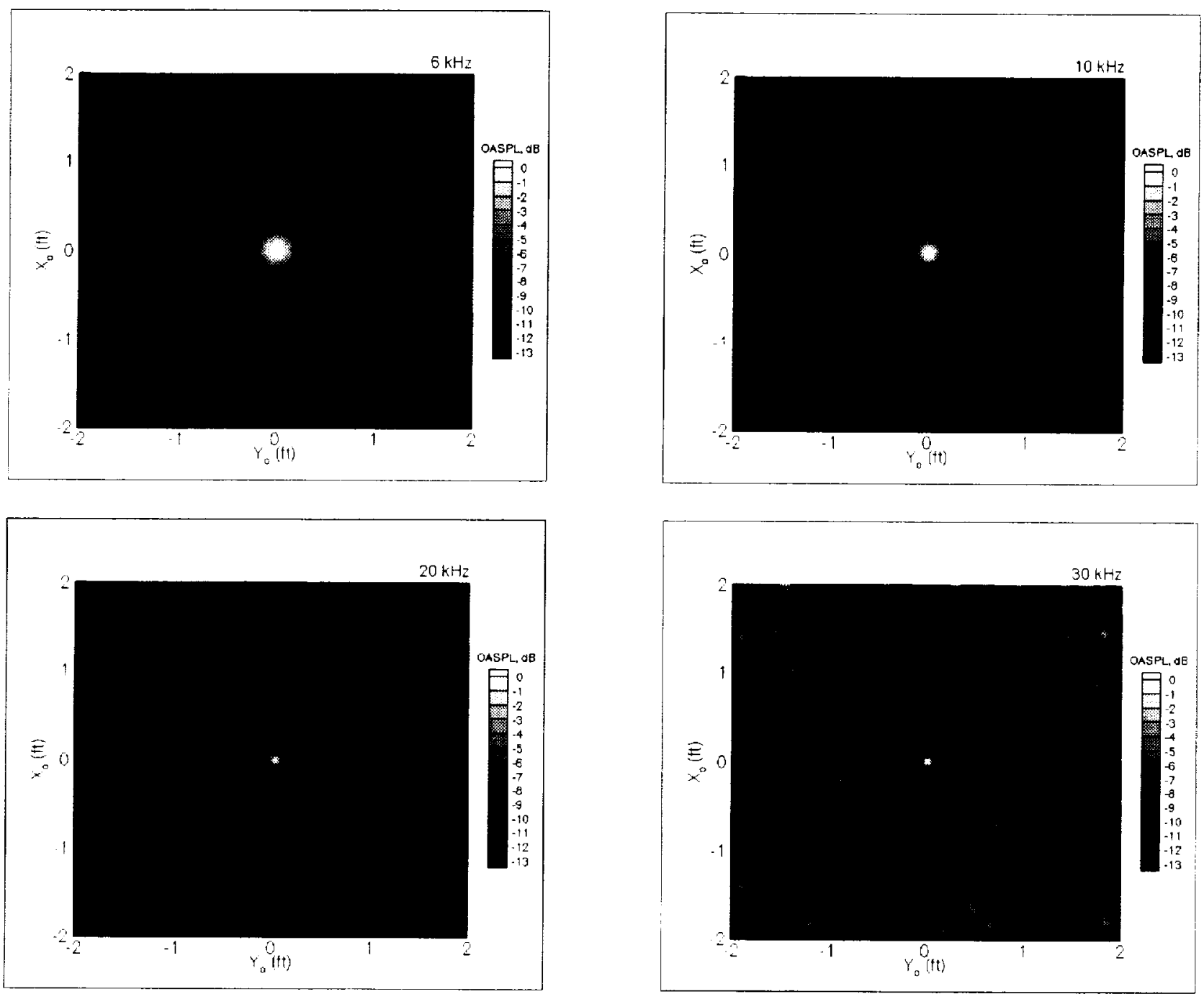

Figure 15. Noise Image Maps from LADA Isolated Point Source Calibration.

Compare with Figure 4.

American Institute of Aeronautics and Astronautics 


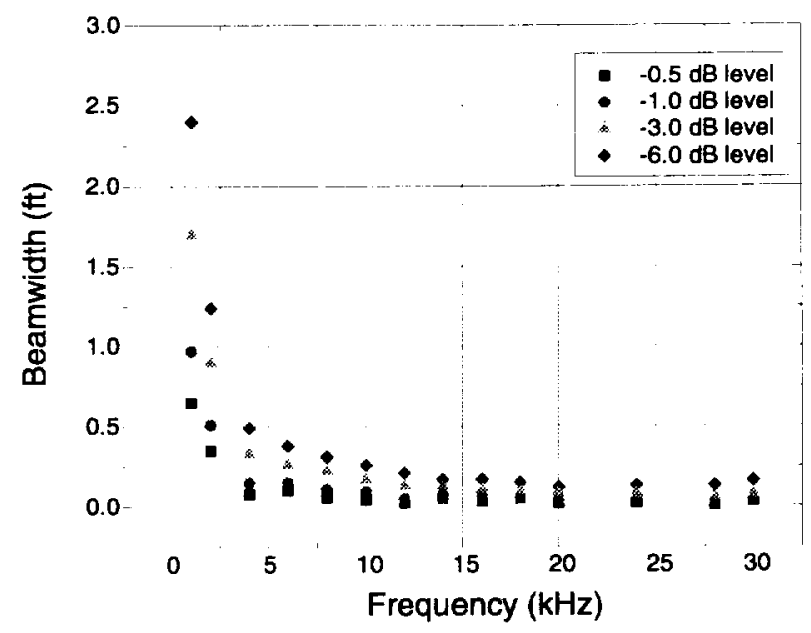

Figure 16. Measured LADA Beamwidth from Isolated Point Source Calibration.

Compare with Figure 5.
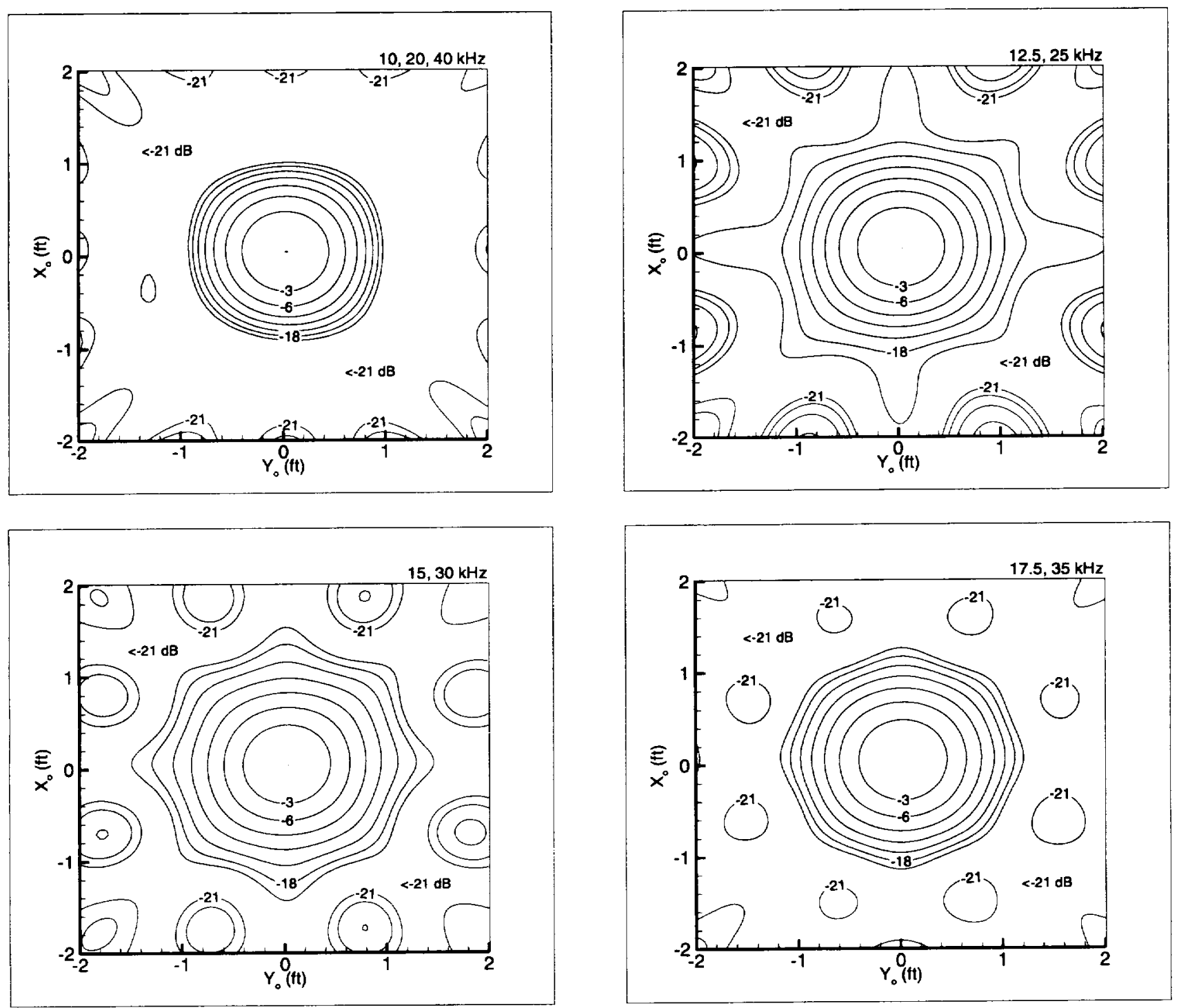

Figure 17. Noise Image Maps from SADA Isolated Point Source Calibration.

Compare with Figure 13. 


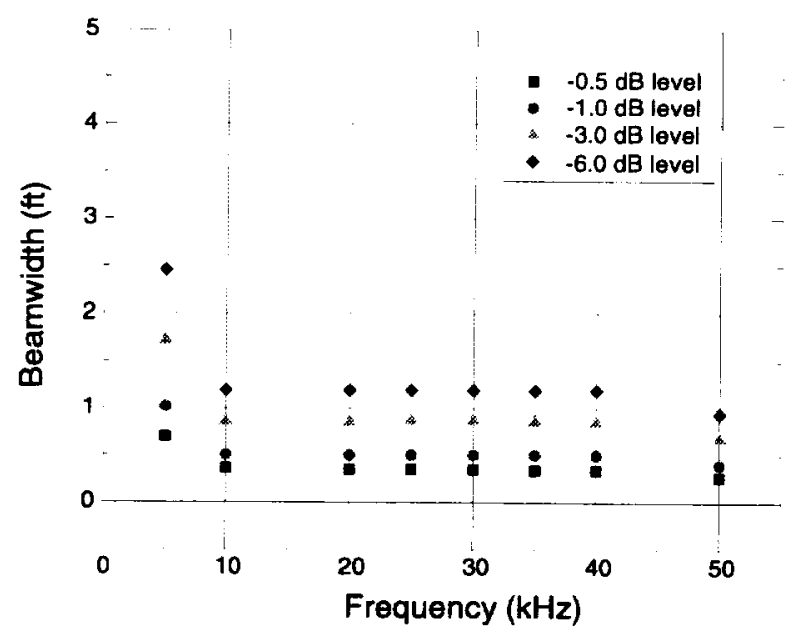

Figure 18. Measured SADA Beamwidths from Isolated Point Source Calibration.

Compare with Figure 14.

(a)
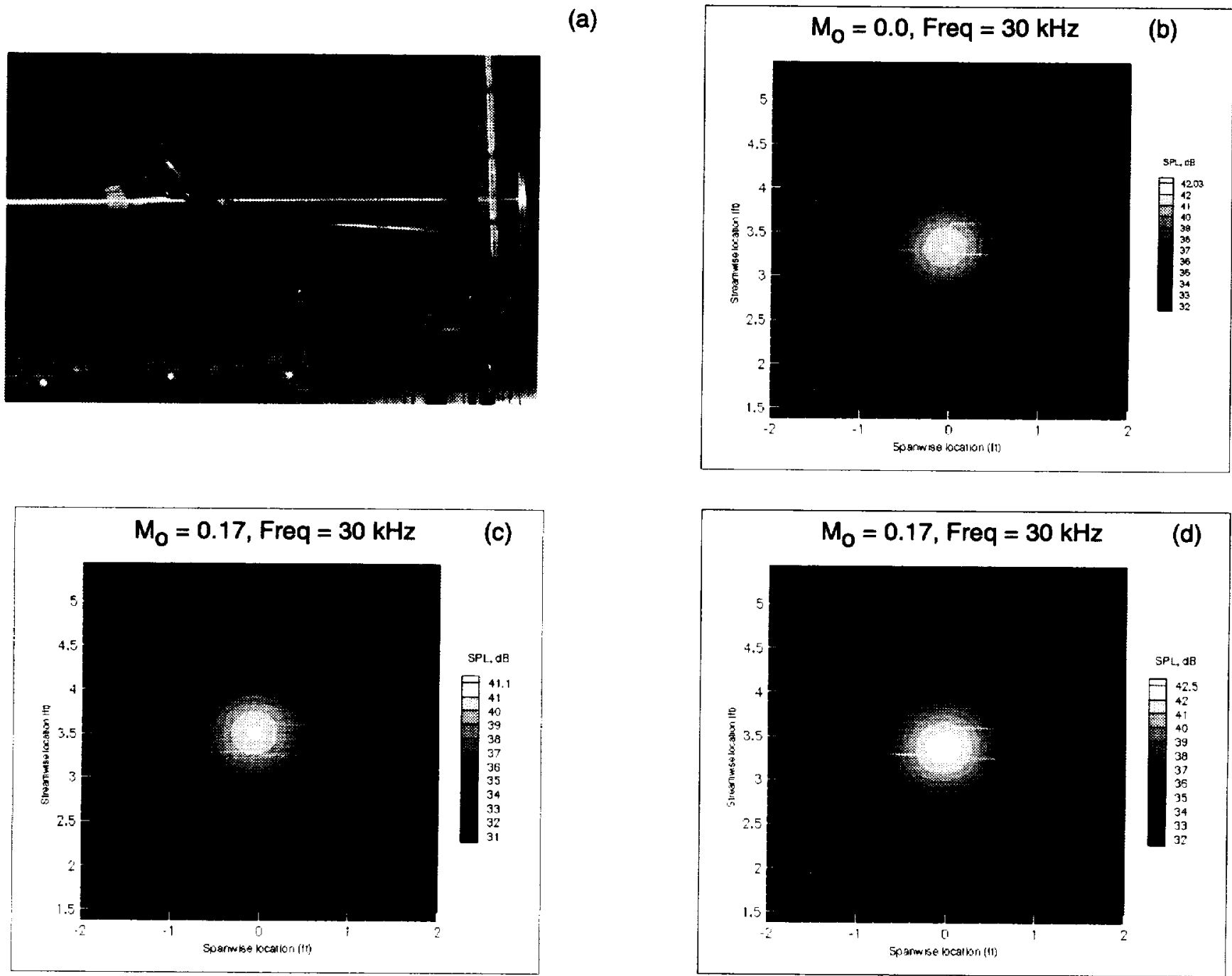

Figure 19. Example of In-Situ Point Source Measurements for Shear Layer Correction Verification.

(a) Photograph of Experiment

(b) Noise Image Map of Point Source With No Flow

(c) Noise Image Map of Point Source - No Shear Correction Applied

(d) Noise Image Map of Point Source with Shear Correction 


$$
M=.17, \alpha=16^{\circ}, \delta_{f}=39^{\circ}
$$
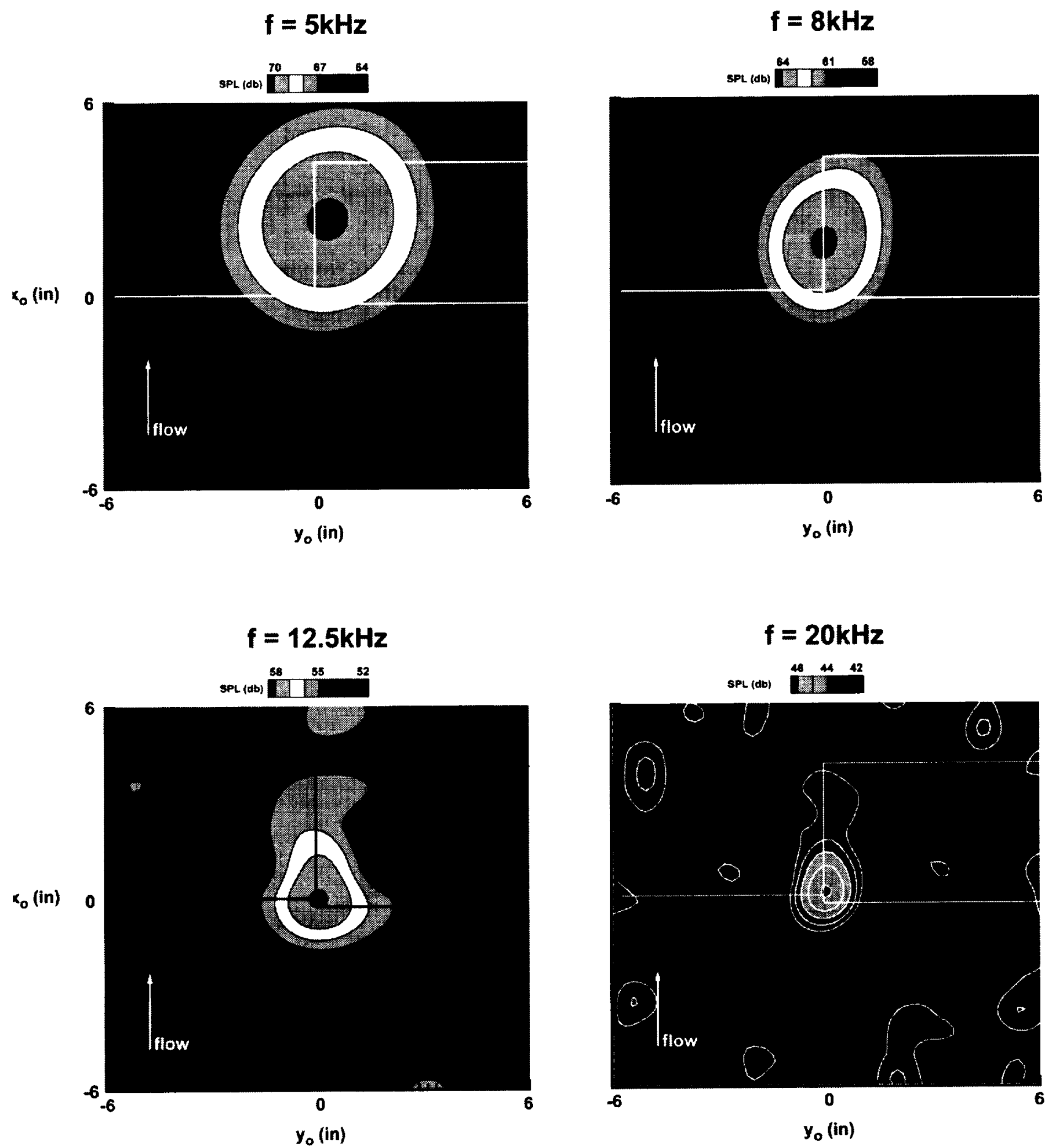

Figure 20. Sound Source Localization Maps for LADA Airframe Noise Model Measurements. $M_{0}=0.17$, Angle-of - Attack=16 deg, Flap=39 deg 


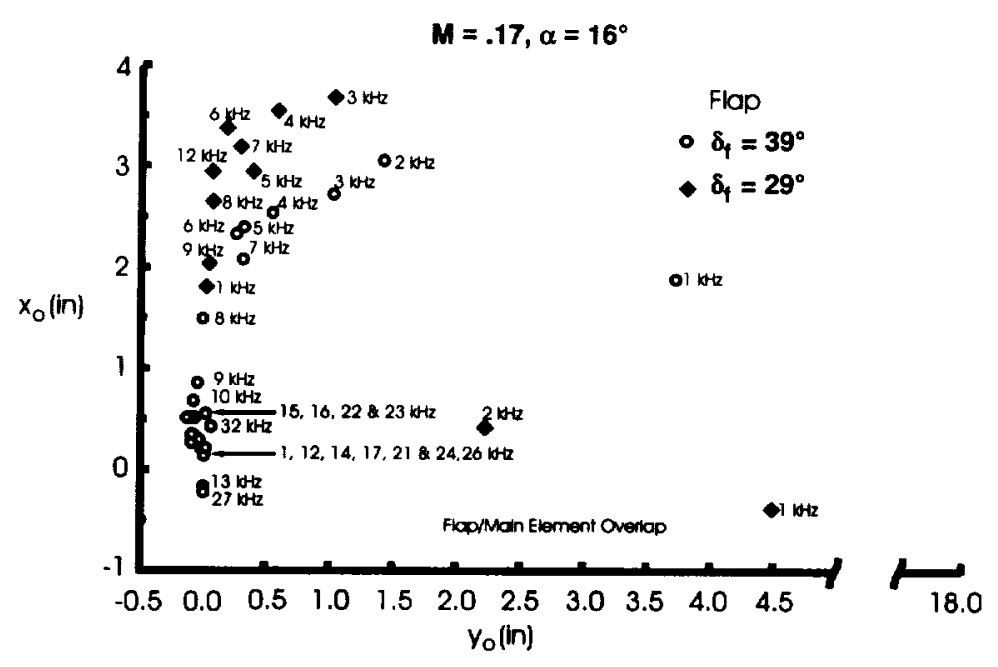

Figure 21. Locally Dominant Noise Source Centroid

Locations on Airframe Noise Model Flap.

Origin Denotes Flap and Main Element Juncture.

$M_{0}=0.17$, Angle-of-Attack $=16 \mathrm{deg}$

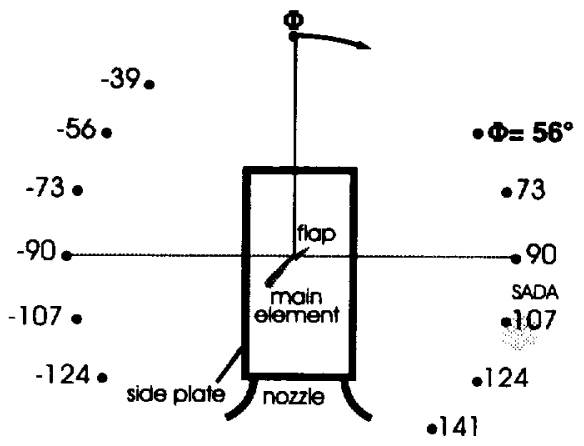

Figure 22. SADA Elevation Angles for Directivity Measurements.

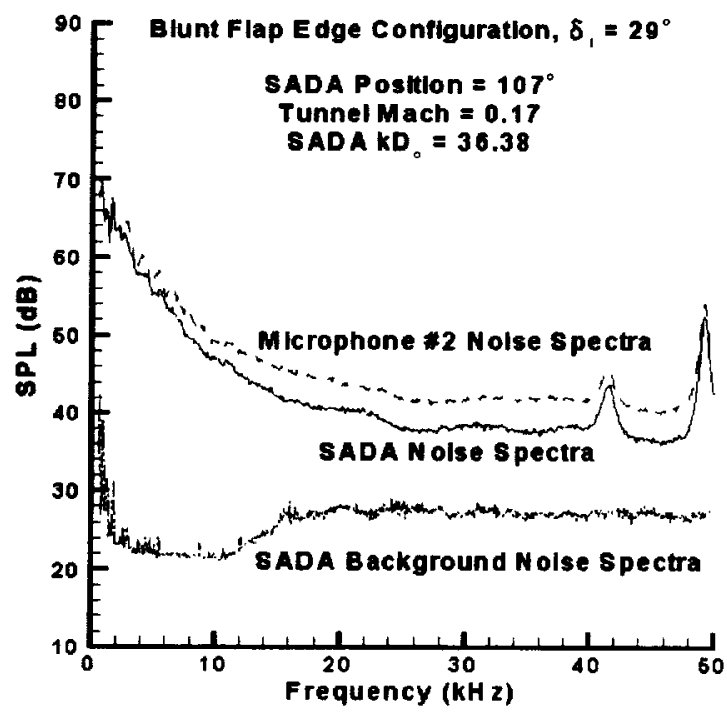

Figure 23. Typical Noise Spectra from SADA Using $87 \mathrm{~Hz}$ Bandwidth. $M_{0}=0.17$, Angle-of-Attack=16 deg, Flap Angle=29 deg

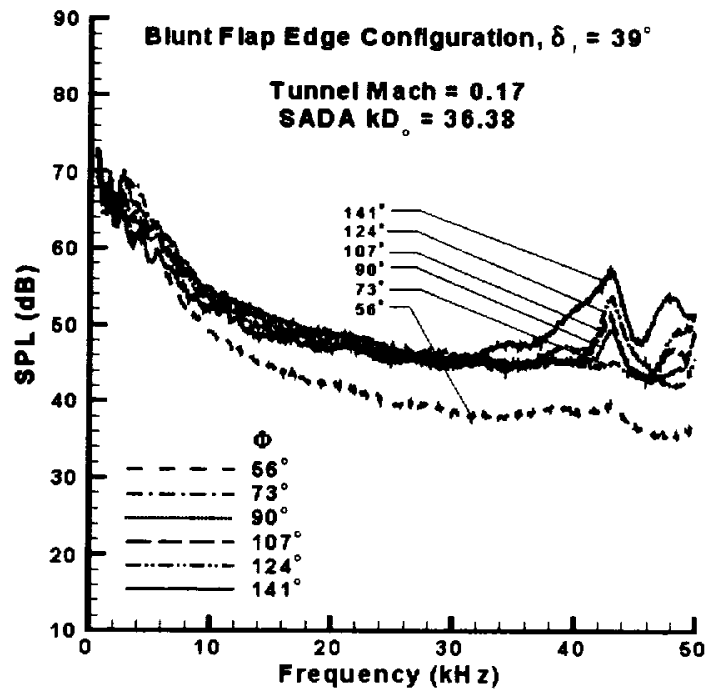

Figure 24. Directivity of Spectra Using $87 \mathrm{~Hz}$ Bandwidth for $M_{0}=0.17$.

Angle-of-Attack=16 deg, Flap Angle $=39 \mathrm{deg}$ 